\title{
LARGE ABELIAN SUBGROUPS OF CHEVALLEY GROUPS
}

\author{
MICHAEL J. J. BARRY
}

(Received 11 January 1978)

Communicated by M. F. Newman

\begin{abstract}
For any group $S$ let $\mathrm{Ab}(S)=\{A \mid A$ is an abelian subgroup of $S$ of maximal order $\}$. Let $G$ be a Chevalley group of type $A_{n}, B_{n}, C_{n}$ or $D_{n}$ over a finite field of characteristic $p$ and let $U \in \mathrm{Syl}_{p}$ $(G)$. In this paper $\mathrm{Ab}(U)$ is determined for all such groups.
\end{abstract}

Subject classification (Amer. Math. Soc. (MOS) 1970): 20 G 40.

\section{Introduction}

Let $q=p^{k}, p$ a prime number. For an odd prime $r$ different from $p$, a theorem of Alperin (1965) shows that an $r$-Sylow subgroup of $\operatorname{GL}(n, q)$ has a unique largest normal abelian subgroup and that no other abelian subgroup has order as great. Goozeff (1970) considered a $p$-Sylow subgroup $U$ of $\operatorname{GL}(n, q)$ where $q$ is odd. He bounded the order of an abelian subgroup of $U$ and showed that this bound is always attained. Goozeff also pointed out that, if $n$ is even, $U$ has a unique largest abelian subgroup. Thwaites (1972) considered a p-Sylow subgroup $U$ of GL $(n, p)$. He showed that if $n$ is even, $U$ contains precisely one abelian subgroup of maximal rank, while if $n$ is odd and $n \geqslant 5, U$ contains precisely two abelian subgroups of maximal rank.

Theorem 2.1 of this paper identifies $\mathrm{Ab}(U)$ where $U$ is a p-Sylow subgroup of $\operatorname{SL}(n, q)$ and hence of $\operatorname{GL}(n, q)$ with no restriction on whether $q$ is even or odd. If $n$ is even $|\mathrm{Ab}(U)|=1$; while if $n$ is odd and $n \geqslant 5,|\mathrm{Ab}(U)|=2$. Finally if $n=3$ then $|\mathrm{Ab}(U)|=q+1$. In all cases $\mathrm{Ab}(U)$ contains groups which are elementary abelian and so the abelian subgroups of maximal rank can be read from the list of groups in $\mathrm{Ab}(U)$. Hence Theorem 2.1 generalizes the results of Goozeff and Thwaites. In Sections 2-5 solutions to the problem for groups of type $B_{n}, C_{n}$ and $D_{n}$ are also 
presented and the results are arranged in order of difficulty. In Section 6 we calculate the Thompson subgroup $J(U)$ of $U$ and we note that $J(U)=\langle A| A \leqslant U, A$ abelian of maximal rank $>$.

Most of the results of this paper were contained in the author's doctoral thesis which was written under the direction of Professor Warren J. Wong at the University of Notre Dame. The author wishes to thank Professor Wong for posing this problem in the first place, and for his patience and encouragement during its solution. Thanks too are owed to the Department of Mathematics at Notre Dame for its generous support throughout.

\section{Notation and terminology}

The current standard references for the theory of Chevalley groups are Steinberg (1968) and Carter (1972). In this section we will fix our notation and dwell a little on those aspects of Chevalley groups which we will need.

$F$ will always denote a finite field of $q=p^{k}$ elements where $p$ is a prime number. Let $\Phi$ be a root system for a simple finite-dimensional Lie algebra $g$ over $C$. Then $\Phi^{+}$will denote the set of positive roots and $\Pi=\left\{r_{1}, r_{2} \ldots, r_{n}\right\}$ the fundamental set relative to some ordering. The universal Chevalley group of type $g$ over $F$, denoted by $g(q)$, is obtained from a particular representation of $g$ over $\mathbf{C}$ by choosing an admissible lattice and 'going mod- $p$ '. Thus $A_{n}(q)$ will mean the universal Chevalley group of type $A_{n}$ over $F$ and the meaning of $B_{n}(q), C_{n}(q)$ and $D_{n}(q)$ is now clear.

Now

$$
\begin{gathered}
A_{n}(q) \cong \operatorname{SL}(n+1, q), \quad C_{n}(q) \cong \operatorname{Sp}(2 n, q), \\
B_{n}(q)=\operatorname{Spin}(2 n+1, q) \text { and } D_{n}(q) \cong \operatorname{Spin}(2 n, q)
\end{gathered}
$$

where Spin $(2 n+1, q)$ (respectively Spin $(2 n, q)$ ), is the universal central extension of $\Omega(2 n+1, q)$ (respectively $\Omega(2 n, q)$ ), the commutator subgroup of $O(2 n+1, q)$ (respectively $O^{+}(2 n, q)$ ) using the notation of Carter (1972), p.6.

Let $G=g(q)$. Then $G=\langle X(r) \mid r \in \Phi\rangle$ where the root subgroup

$$
X(r)=\langle x(r, t) \mid t \in F\rangle \cong F
$$

as an additive group. We define the subgroup $U=\left\langle X(r) \mid r \in \Phi^{+}\right\rangle$. Now $U$ is a $p$-Sylow subgroup of $G$. We shall be concerned with $\operatorname{Ab}(U)$.

Let $B$ be the normalizer of $U$ in $G$. Then $B$ is a semi-direct product of $U$ with $H$ where $H$ is an abelian $p^{\prime}$-group. The parabolic subgroups of $G$ are those subgroups which contain $B$ and there is a natural bijection between the family of subsets of $\Pi$ and the parabolic subgroups of $G$.

Theorem 1.1. Suppose $G$ is a Chevalley group amd $P_{J}$ the parabolic subgroup naturally associated with $J \subseteq \Pi$. Then $P_{J}=L_{J} U_{J}$, a semidirect product with $U_{J} \preccurlyeq P_{J}$, is 
known as the Levi decomposition of $P_{J}$. Here $U_{J}=\left\langle X(r) \mid r \in \Phi^{+}-\Phi_{J}\right\rangle, L_{J}=\left\langle H, S_{J}\right\rangle$, $S_{J}=\left\langle X(r) \mid r \in \Phi_{J}\right\rangle$ and $\Phi_{J}$ is the root system with $J$ as the fundamental set.

We next define a partial order on $\Phi$ as follows: if $r, s \in \Phi$ then $r \geqslant s$ if $r-s$ is a non-negative linear integral combination of elements of $\Pi$.

Examples. In Theorem 1.1, if $J=\Pi-\left\{s_{1}, \ldots, s_{r}\right\}$ then $U_{J}=\langle X(r)| r \geqslant s_{j}$ for some $j$ such that $1 \leqslant j \leqslant r\rangle$.

Throughout this paper, the Dynkin diagrams of the indecomposable roots systems of type $A_{n}, B_{n}, C_{n}$, and $D_{n}$ will be labelled as in Humphreys (1972), p. 58.

The root system $\Phi$ can be thought of as a subset of $\mathbf{R}^{n}$ where $\Pi=\left\{r_{1}, r_{2}, \ldots, r_{n}\right\}$ is a basis for $\mathbf{R}^{n}$. If $\mathbf{R}^{n}$ is equipped with an inner product (,) we define a new form $\left\langle_{,}\right\rangle$on $\mathbf{R}^{n}$ as follows:

$$
\langle r, s\rangle=2(r, s) /(s, s)
$$

for all $r, s \in \mathbf{R}^{n}, s \neq 0$. A vector $\lambda \in \mathbf{R}^{n}$ is called an abstract weight provided that $\langle\lambda, r\rangle$ is integral for all $r \in \Phi$. These vectors form a lattice $\Lambda$ which has a basis of fundamental dominant weights $\left\{\lambda_{1}, \lambda_{2}, \ldots, \lambda_{n}\right\}$ for which $\left\langle\lambda_{l}, r_{j}\right\rangle=\delta_{i j}$.

A dominant weight is any non-negative linear integral combination of the $\lambda_{i}$, $1 \leqslant i \leqslant n$. Denote by $\Lambda^{+}$the set of all dominant weights. For each $\lambda \in \Lambda^{+}$there is (to within isomorphism) exactly one irreducible $g$-module $V(\lambda)$ over $C$ whose highest weight is $\lambda$ and this weight occurs with multiplicity 1 . By choosing an admissible lattice and 'going mod-p' we construct a $g(q)$-module over $F$ which we denote by $V(g, n, \lambda)$ where $n$ is the rank of $g$.

2. The solution for $A_{n}(q)$, any $q$, and $C_{n}(q), q$ odd

THEOREM 2.1.

(a) Let $G=A_{2 n+1}(q)$ and $B=\left\langle X(r) \mid r \geqslant r_{n+1}\right\rangle$ Then $\mathrm{Ab}(U)=\{B\}$ and $|B|=q^{(n+1)^{2}}$.

(b) Let $G=A_{2 n}(q), B(1)=\left\langle X(r) \mid r \geqslant r_{n}\right\rangle$ and $B(2)=\left\langle X(r) \mid r \geqslant r_{n+1}\right\rangle$. Then

(i) if $n>1, \mathrm{Ab}(U)=\{B(1), B(2)\}$;

(ii) if $n=1, \mathrm{Ab}(U)=\left\{B(1), B(2), B(\mathrm{a}) \mid a \in F^{*}\right\}$,

where $B(a)=\left\langle x\left(r_{1}, t\right) x\left(r_{2}, a t\right), X\left(r_{1}+r_{2}\right) \mid t \in F\right\rangle$. Further, any element of $\mathrm{Ab}(U)$ has order $q^{n(n+1)}$.

Proof. We will prove only $(b)$ from which the method of proving $(a)$ will be quite apparent. We proceed by induction on $n$.

Consider $n=1$. Then $U=\left\langle X\left(r_{1}\right), X\left(r_{2}\right), X\left(r_{1}+r_{2}\right)\right\rangle$. If $A \in \mathrm{Ab}(U)$ then clearly $Z(U)=X\left(r_{1}+r_{2}\right) \leqslant A$. Now

$$
C_{U}\left(x\left(r_{1}, t\right)\right)=\left\langle X\left(r_{1}\right), X\left(r_{1}+r_{2}\right)\right\rangle
$$


and

$$
C_{v}\left(x\left(r_{1}, a t\right) x\left(r_{2}, t\right)\right)=\left\langle x\left(r_{1}, a s\right) x\left(r_{2}, s\right), X\left(r_{1}+r_{2}\right) \mid s \in F\right\rangle \text { if } t \neq 0 .
$$

Gathering these pieces of information together and noting that these centralizers are abelian we get part (ii) of (b).

We now assume the result is true for any integer $r$ where $1 \leqslant r<n$. Let $P_{J}$ be the parabolic subgroup of $G=A_{2 n}(q)$ associated with $J=\Pi-\left\{r_{1}, r_{2 n}\right\}$. Then

$$
S_{J}=\left\langle X(r) \mid r \in \Phi_{j}\right\rangle \cong A_{2 n-2}(q)
$$

and

$$
\left.U_{J}=\langle X(r)| r \geqslant r_{1} \text { or } r \geqslant r_{2 n}\right\rangle \text {. }
$$

Further, $\left[U_{J}, U_{J}\right]=X\left(r_{0}\right)=Z\left(U_{J}\right)$ where $r_{0}=r_{1}+r_{2}+\ldots+r_{2 n}$ is the highest root. From now on let $V=U_{J}$. Let $U_{1}=\left\langle X(r) \mid r \in \Phi_{J}^{+}\right\rangle$. Then $U_{1} \in \operatorname{Syl}_{p}\left(S_{J}\right)$ and by induction.

$$
\begin{aligned}
\operatorname{Ab}\left(U_{1}\right) & =\left\{B_{1}(1), B_{1}(2)\right\} \quad \text { if } n>2 \\
& =\left\{B_{1}(1), B_{1}(2), B_{1}(a) \mid a \in F^{*}\right\} \quad \text { if } n=2,
\end{aligned}
$$

where

$$
\begin{aligned}
& B_{1}(1)=\left\langle X(r) \mid r \in \Phi_{J}^{+}, r \geqslant r_{n}\right\rangle, \\
& B_{2}(2)=\left\langle X(r) \mid r \in \Phi_{J}^{+}, r \geqslant r_{n+1}\right\rangle
\end{aligned}
$$

and

$$
B_{2}(a)=\left\langle x\left(r_{2}, t\right) x\left(r_{3}, a t\right), X\left(r_{2}+r_{3}\right) \mid t \in F\right\rangle
$$

LeMma 2.2. If $C \in \mathrm{Ab}(V)$, then $|C|=q^{2 n}$.

PROOF. Let $K=\left\{r \in \Phi^{+} \mid r \neq r_{0}, r \geqslant r_{1}\right.$ or $\left.r \geqslant r_{2 n}\right\}$ and let ' be the natural epimorphism of $V$ on $V \mid Z(V)=V^{\prime}$. Then $V^{\prime}$ is abelian since $Z(V)=[V, V]$. Now $V^{\prime}$ can be made into a vector space over $F$ with basis $\left\{x^{\prime}(r, 1) \mid r \in K\right\}$ by defining

$$
t x^{\prime}(r, 1)+u x^{\prime}(s, 1)=x^{\prime}(r, t) x^{\prime}(a, u)
$$

for all $r, s \in K, t, u \in F$.

Moreover it is even possible to equip $V^{\prime}$ with an alternating bilinear form (,) as follows:

$$
x\left(r_{0},\left(v_{1}^{\prime}, v_{2}^{\prime}\right)\right)=\left[v_{1}, v_{2}\right]
$$

for all $v_{1}^{\prime}, v_{2}^{\prime} \in V^{\prime}$. Note this definition is independent of the choices of the preimages $v_{1}, v_{2}$ in $V$ since the kernel of ${ }^{\prime}=Z(V)=[V, V]$. The form is non-degenerate since $\left(v_{1}^{\prime}, v^{\prime}\right)=0$ for all $v^{\prime} \in V^{\prime}$ implies $\left[v_{1}, v\right]=1$ for all $v \in V$ which implies $v_{1} \in Z(V)$ and finally that $v_{1}^{\prime}=0$.

It is clear that a subgroup $W$ of $V$ is abelian if and only if $(W)^{\prime}$ is contained in a totally isotropic subspace of $V^{\prime}$. The maximal dimension of a totally isotropic subspace 
of $V^{\prime}=\operatorname{dim} V^{\prime} / 2=2 n-1$. Therefore $W$ abelian implies that $\left|\mathrm{W}^{\prime}\right| \leqslant q^{2 n-1}$ and hence $|W| \leqslant q^{2 n}$. In fact $\left\langle X(r) \mid r \geqslant r_{1}\right\rangle$ is an abelian subgroup of $V$ of order $q^{2 n}$. This completes the proof of Lemma 2.2 .

Let

$$
\left.V_{1}(1)=\langle X(r)| r \geqslant r_{1}+\ldots+r_{n} \text { or } r \geqslant r_{n}+\ldots+r_{2 n}\right\rangle
$$

and

$$
\left.V_{1}(2)=\langle X(r)| r \geqslant r_{1}+\ldots+r_{n+1} \text { or } r \geqslant r_{n+1}+\ldots+r_{2 n}\right\rangle
$$

LEMMA 2.3.

(a) $V_{1}(i) \triangleleft U, i=1,2$.

(b) $V_{1}(i) \in \mathrm{Ab}(V)$ and hence $C_{V}\left(V_{1}(i)\right)=V_{1}(i), i=1,2$.

(c) $C_{V}\left(B_{1}(i)\right)=\left(V_{1}(i)\right)^{\prime}, i=1,2$.

(d) $\left[B_{1}(i), V_{1}(i)\right]=1$ and $B(i)=B_{1}(i) V_{1}(i), i=1,2$.

Proof.

(a) This follows from Chevalley's commutator formula.

(b) $V_{1}(i)$ is abelian, $\left|V_{1}(i)\right|=q^{2 n}$ and so $V_{1}(i) \in \mathrm{Ab}(V)$ by Lemma 2.2. Hence $C_{v}\left(V_{1}(i)\right)=V_{1}(i), i=1,2$.

(c) This follows by inspection.

(d) This follows from the commutator formula and the definitions of $B(i), B_{1}(i)$ and $V_{1}(i), i=1,2$.

This completes the proof of Lemma 2.3.

Let $\varphi$ be the natural epimorphism of $U$ on $U / V \cong U_{1}$, and let $A \in \mathrm{Ab}(U)$. Then $|A| \geqslant q^{n(n+1)}=|B(i)|, \quad i=1,2$. Further, $(A) \varphi=A_{1} \leqslant U_{1}$ and $|A|=\left|A_{1}\right||A \cap V|$. Therefore

$$
|B(i)| \leqslant|A|=\left|A_{1}\right||A \cap V| \leqslant\left|B_{1}(i)\right| q^{2 n}=\left|B_{1}(i)\right|\left|V_{1}(i)\right|=|B(i)|, \quad i=1,2,
$$

making use of Lemmas 2.2 and 2.3. Hence $A_{1} \in \mathrm{Ab}\left(U_{1}\right)$ and $A \cap V \in \mathrm{Ab}(V)$. Calling on our induction hypothesis $A_{1} \in \mathrm{Ab}\left(U_{1}\right)$ means that $A_{1}=B_{1}(1)$ or $B_{1}(2)$ if $n>2$ and $A_{1}=B_{1}(1)$ or $B_{1}(2)$ or $B_{1}(a)$ if $n=2$.

We consider the case $n=2$ and $A_{1}=B_{2}(a)$ for some $a \in F^{*}$. Clearly

$$
(A \cap V)^{\prime} \leqslant C_{V}^{\prime}\left(B_{1}(a)\right) \text {. }
$$

An easy calculation gives $C_{V},\left(B_{1}(a)\right)=\left\langle X^{\prime}\left(r_{1}+r_{2}+r_{3}\right), X^{\prime}\left(r_{2}+r_{3}+r_{4}\right)\right\rangle$. This implies $\left|(A \cap V)^{\prime}\right| \leqslant q^{2}$ and hence $|A \cap V| \leqslant q^{3}$. This contradicts $A \cap V \in \mathrm{Ab}(V)$ and so we have ruled out the case $A_{1}=B_{1}(a)$ for some $a \in F$. Therefore $A_{1}=B_{1}(1)$ or $B_{1}(2)$ in all cases.

We may suppose that $A_{1}=B_{1}(1)$. Then $A \leqslant B_{1}(1) V$. Note that $B_{1}(1) \cap V=1$. 
Let $v \in A \cap V, a \in A$. Then $a=b v_{1}$ where $b \in B_{1}(1)$ and $v_{1} \in V$. Now

$$
1=[a, v]=[b, v]^{v_{1}}\left[v_{1}, v\right] \text {. }
$$

Hence $[b, v]^{v_{1}} \in Z(V)=[V, V]$ and hence $[b, v] \in Z(V)$.

Now since $A_{1}=B_{1}(1)$, given any $b \in B_{1}(1)$ there exists $a \in A$ such that $a=b w$ for some $w \in V$. Therefore $\left[B_{1}(1), v\right] \leqslant Z(V)$ and so $v^{\prime} \in C_{V} \cdot\left(B_{1}(1)\right)$. By Lemma 2.3(c) $v \in V_{1}(1)$ and so $A \cap V \leqslant V_{1}(1)$. Since $|A \cap V|=\left|V_{1}(1)\right|$ we get equality.

Again let $a \in A, a=b v_{1}$ where $b \in B_{1}(1)$ and $v_{1} \in V$ and let $v \in A \cap V=V_{1}(1)$. Then

$$
1=[a, v]=[b, v]^{v_{1}}\left[v_{1}, v\right]=\left[v_{1}, v\right]
$$

since $\left[B_{1}(1), V_{1}(1)\right]=1$ by Lemma $2.3(\mathrm{~d})$. Therefore $v_{1} \in C_{V}\left(V_{1}(1)\right)=V_{1}(1)$ by Lemma 2.3(b). We have proved $A \leqslant B_{1}(1) V_{1}(1)=B(1) .|A| \geqslant|B(1)|$ gives equality. This completes the proof of Theorem 2.1 .

NoTE 2.4. Two techniques in this proof will be used again and again. Firstly $J$ will always be chosen so that $\left[U_{J}, U_{J}\right]=X\left(r_{0}\right) \leqslant Z\left(U_{J}\right)$ where $r_{0}$ is the highest root and $Z\left(U_{J}\right)$ is the direct product of root subgroups. In this way by putting a non-degenerate alternating form on $U_{\mathrm{J}} / Z\left(U_{\mathrm{J}}\right)$ we will always be able to get an exact bound on the elements of $\mathrm{Ab}\left(U_{J}\right)$. Secondly, whenever we have a situtaion where $A \in \operatorname{Ab}(U)$, we have identified $A_{1}$ and $A \cap U_{J}$, and we can compute that $A \cap U_{J} \in \mathrm{Ab}\left(U_{J}\right),\left[A_{1}, A \cap U_{J}\right]$ $=1$ and that the centralizer of $A_{1}$ in $U_{J}^{\prime}$ is $\left(A \cap U_{J}\right)^{\prime}$ where ' maps $U_{J}$ naturally onto $U_{J} / X\left(r_{0}\right)$, then we shall be able to prove that $A=A_{1} \times\left(A \cap U_{J}\right)$ exactly as we did towards the end of the above proof.

THEOREM 2.5. Let $G=C_{n}(q)$ where $n \geqslant 2$ and $q$ is odd. Let $B=\left\langle X(r) \mid r \geqslant r_{n}\right\rangle$. Then $\operatorname{Ab}(U)=\{B\}$ and $|B|=q^{n(n+1) / 2}$.

Proof. The proof is by induction on $n$. If $n=2$,

$$
B=\left\langle X\left(r_{2}\right), X\left(r_{1}+r_{2}\right), X\left(2 r_{1}+r_{2}\right)\right\rangle
$$

and $|B|=q^{3}$. In Wong (1969) it is established that in $P S p(4, q), q$ odd, and hence in $C_{2}(q), U$ contains a unique largest abelian subgroup of order $q^{3}$. Hence $B$ is this subgroup and the result is true for $n=2$.

We now assume the result for any integer $r$ where $2 \leqslant r<n$. Let $P_{J}$ be the parabolic subgroup of $G=C_{n}(q)$ associated with $J=\Pi-\left\{r_{1}\right\}$. Then $S_{J}=\left\langle X(r) \mid r \in \Phi_{J}\right\rangle \cong$ $C_{n-1}(q)$, and $U_{J}=\left\langle X(r) \mid r \geqslant r_{1}\right\rangle$. Further, $\left[U_{J}, U_{J}\right]=X\left(r_{0}\right)=Z\left(U_{J}\right)$ where $r_{0}=$ $2 r_{1}+2 r_{2}+\ldots+2 r_{n-1}+r_{n}$ is the highest root. Note that if $q$ is even $\left[U_{J}, U_{J}\right]=1$. From now on let $V=U_{J}$.

Let $U_{1}=\left\langle X(r) \mid r \in \Phi_{J}^{+}\right\rangle$. Then $U_{1} \in \operatorname{Syl}_{p}\left(S_{J}\right)$ and by induction $\operatorname{Ab}\left(U_{1}\right)=\left\{B_{1}\right\}$ where $B_{1}=\left\langle X(r) \mid r \in \Phi_{J}^{+}, r \geqslant r_{n}\right\rangle$. Finally let $V_{1}=\left\langle X(r) r \geqslant r_{1}+r_{2}+\ldots+r_{n}\right\rangle$. Then using the techniques of Theorem 2.1 one forces, for any $A \in \mathrm{Ab}(U)$, that $A_{1}=B_{1}$ and $A \cap V=V_{1}$ and finally that $A=B_{1} V_{1}=B$. Hence Theorem 2.5.

What about $C_{n}(q), q$ even? This will be attended to in Section 4. 


\section{The solution for $D_{n}(q)$}

Theorem 3.1. Let $G=D_{4}(q)$. Then $A \in \mathrm{Ab}(U)$ implies $|A|=q^{6}$. Further,

(a) if $q$ is odd, $\mathrm{Ab}(U)=\left\{B_{1}, B_{2}, B_{3}\right\}$ where $B_{1}=\left\langle X(r) \mid r \geqslant r_{1}\right\rangle, B_{2}=\left\langle X(r) \mid r \geqslant r_{3}\right\rangle$ and $B_{3}=\left\langle X(r) \mid r \geqslant r_{4}\right\rangle$;

(b) if $q$ is even and $q \geqslant 4, \mathrm{Ab}(U)=\{B(1, a), B(2, b), B(3, c) \mid a, b, c \in F\}$ where

$$
\begin{gathered}
B(1, a)=\left\langle x\left(r_{1}, t_{1}\right) x\left(r_{3}, a t_{1}\right), x\left(r_{1}+r_{2},{ }_{2}\right) x\left(r_{2}+r_{3}, a t_{2}\right), x\left(r_{1}+r_{2}+r_{4}, t_{3}\right)\right. \\
x\left(r_{2}+r_{3}+r_{4}, a t_{3}\right), X(r)\left|r \geqslant r_{1}+r_{2}+r_{3}, t_{i} \in F\right\rangle
\end{gathered}
$$

and $B(2, c)$ and $B(3, c)$ are defined in a similar fashion;

(c) if $q=2, \mathrm{Ab}(U)$ is as in (b) with one additional element, namely $A^{*}$ which is defined in Lemma 3.10.

THEOREM 3.2. Let $G=D_{n}(q)$ where $n \geqslant 5$. Then $A \in \mathrm{Ab}(U)$ implies $|A|=q^{n(n-1) / 2}$. Further,

(a) if q is odd, $\mathrm{Ab}(U)=\left\{B_{1}, B_{2}\right\}$ where $B_{1}=\left\langle X(r) \mid r \geqslant r_{n-1}\right\rangle$ and $B_{2}=\left\langle X(r) \mid r \geqslant r_{n}\right\rangle$

(b) if $q$ is even, $\mathrm{Ab}(U)=\left\{B_{2}, B(1, a) \mid a \in F\right\}$ where $B_{2}$ is as in (a) and

$$
B(1, a)=\left\langle x(r, t) x(\bar{r}, a t), X(s) \mid r \geqslant r_{n-1}, r \neq r_{n-2}+r_{n-1}+r_{n}, s \geqslant r_{n-2}+r_{n-1}+r_{n}, t \in F\right\rangle,
$$

where $\bar{r}_{1}=r_{1}, \bar{r}_{2}=r_{2}, \ldots, \bar{r}_{n-2}=r_{n-2}, \bar{r}_{n-1}=r_{n}, \bar{r}_{n}=r_{n-1}$ and - is extended by linearity to $\Phi$.

Before we attempt the proofs of Theorems 3.1 and 3.2 we establish some notation for this section and we prove some general results. Let $P_{J}$ be the parabolic subgroup of $G$ associated with $J=\Pi-\left\{r_{2}\right\}$. Then, if necessary, by Barry (1977), Theorem 3.2 we have $S_{J}=M \times N$ where $M=\left\langle X\left(r_{1}\right), X\left(-r_{1}\right)\right\rangle \cong A_{1}(q)$ and $N=\left\langle X(r) \mid r \in \Phi_{K}\right\rangle \cong$ $D_{n-2}(q)$ where $K=\Pi-\left\{r_{1}, r_{2}\right\}$ Also $U_{J}=\left\langle X(r) \mid r \geqslant r_{2}\right\rangle$ and $\left[U_{J}, U_{J}\right]=X\left(r_{0}\right)=Z\left(U_{J}\right)$ where $r_{0}=r_{1}+2 r_{2}+\ldots+2 r_{n-2}+r_{n-1}+r_{n}$ is the highest root. From now on let $V=U_{\mathrm{J}} . M \times N$ acts on $V$ by right conjugation and by Barry (1977), Theorem 3.2 we have

and

$$
V /[V, V] \cong V\left(A, 1, \lambda_{1}\right) \otimes W \text { as an } M \times N \text {-module }
$$

$$
V /[V, V] \cong W \otimes W \text { as an } N \text {-module, }
$$

where $W=V\left(D, n-2, \lambda_{1}\right)$ can be identified with $\left\langle X(r) \mid r \geqslant r_{2}, r \neq r_{1}\right\rangle$.

Let $X=X\left(r_{1}\right)$ and $Y=\left\langle X(r) \mid r \in \Phi_{K}^{+}\right\rangle$. Then $X \times Y \in \operatorname{Syl}_{p}(M \times N)$ and $U=X Y V$. Let $\varphi$ be the natural epimorphism of $U$ on $U / V$ and ' that of $V$ on $V /[V, V]$.

LEMMA 3.3. Let $x \in X$ and $y \in Y$. Let $\{e, f\}$ be a basis of $V\left(A, 1, \lambda_{1}\right)$ such that $e x=e$ and $f x=f+a e$. Then

(1) if $x \neq 1, a \neq 0$; further, $e \otimes u+f \otimes v \in C_{V},(x y)$ if and only if $u \in \operatorname{ker}_{W}(y-1)^{2}$ and $v=-u(y-1) / a$ where $u, v \in W$ and so $\operatorname{dim} C_{V},(x y)=\operatorname{dim} \operatorname{ker}_{W}(y-1)^{2}$.

(2) $e \otimes u+f \otimes v \in C_{V},(y)$ if and only if $u, v \in C_{W}(y)$. 
Proof.

(1) $V\left(A, 1, \lambda_{1}\right)$ is the natural representation for $A(q) \cong \operatorname{SL}(2, q)$ and hence is faithful. Therefore if $x \neq 1, a \neq 0$.

Let $e \otimes u+f \otimes v \in C_{V},(x y)$ where $x \neq 1$. Then

$$
\begin{aligned}
e \otimes u+f \otimes v & =(e \otimes u+f \otimes v) x y . \\
& =e \otimes u y+(f+a e) \otimes v y \\
& =e \otimes(u y+a v y)+f \otimes v y .
\end{aligned}
$$

Uniqueness of expression gives $v y=v$ and $u=u y+a v \dot{y}$. Therefore $v(y-1)=0$ and $v=-u(y-1) / a$. So $0=v(y-1)=-u(y-1)^{2} / a$ gives us that $u \in \operatorname{ker}_{W}(y-1)^{2}$. The result in the other direction is trivial. Since $u$ determines $v$ and $u \in \operatorname{ker}_{W}(y-1)^{2}$ we have $\operatorname{dim} C_{V},(x y)=\operatorname{dim} \operatorname{ker}_{W}(y-1)^{2}$.

(2) Trivial.

We record

LEMMA 3.4. If $C \in \mathrm{Ab}(V)$ then $|C|=q^{2 n-3}$.

REMARK 3.5. Note that $V=V_{1} V_{2}$ where $V_{1}=\left\langle X(r) \mid r \geqslant r_{1}+r_{2}\right\rangle \Delta U$ and $V_{2}=$ $\left\langle X(r) \mid r \geqslant r_{2}, r \neq r_{1}\right\rangle$. Let $x \in X, y \in Y$ and $v_{i} \in V_{i}, i=1,2$. Then

$$
\begin{aligned}
{\left[x y, v_{1} v_{2}\right] } & =\left[x, v_{2}\right]^{y}\left[x, v_{1}\right]^{v_{2} y}\left[y, v_{2}\right]\left[y, v_{1}\right]^{v_{2}} \\
& =\left[x, v_{2}\right]^{y}\left[y, v_{2}\right]\left[y, v_{1}\right]^{v_{2}}
\end{aligned}
$$

since $\left[x, v_{1}\right]=1$. If $\left[x y, v_{1} v_{2}\right] \in X\left(r_{0}\right)$ then $\left[y, v_{2}\right]=1$ and hence

$$
\left[x y, v_{1} v_{2}\right]=\left[x, v_{2}\right]\left[y, v_{1}\right]^{v_{2}}
$$

since $x^{y}=x$ and $v_{2}^{y}=v_{2}$.

Proof of Theorem. 3.1. This will follow in a series of lemmas.

LEMMA 3.6. Let $x=x\left(r_{1}, t_{1}\right), y=x\left(r_{3}, a t_{1}\right), t_{1} \neq 0$. Then

$$
\begin{aligned}
& C_{V},(x y)= \\
& \left\langle x^{\prime}\left(r_{1}+r_{2}, d_{1}\right) x^{\prime}\left(r_{2}+r_{3},-N\left(r_{3}, r_{1}+r_{2}\right) N\left(r_{1}, r_{2}+r_{3}\right) \mathrm{ad}_{1}\right),\right. \\
& x^{\prime}\left(r_{1}+r_{2}+r_{4}, d_{2}\right) x^{\prime}\left(r_{2}+r_{3}+r_{4},-N\left(r_{3}, r_{1}+r_{2}+r_{4}\right) N\left(r_{1}, r_{2}+r_{3}+r_{4}\right) \mathrm{ad}_{2}\right), \\
& X^{\prime}\left(r_{1}+r_{2}+r_{3}\right), X^{\prime}\left(r_{1}+r_{2}+r_{3}+r_{4}\right)\left|d_{i} \in F, i=1,2\right\rangle .
\end{aligned}
$$

Proof. Let $v_{1} v_{2}$ be an element of the preimage of $C_{V},(x y)$ in $V$ where $v_{i} \in V_{i}$, $i=1,2$. Then $v_{2}=x\left(r_{2}+r_{3}, u_{1}\right) x\left(r_{2}+r_{3}+r_{4}, u_{2}\right)$ by Remark 3.5.

Suppose

$$
v_{1}=x\left(r_{1}+r_{2}, d_{1}\right) x\left(r_{1}+r_{2}+r_{3}, d_{2}\right) x\left(r_{1}+r_{2}+r_{4}, d_{3}\right) x\left(r_{1}+r_{2}+r_{3}+r_{4}, d_{4}\right) .
$$


We are ignoring any component of $Z(V)$ in $v_{1}$. Then

$$
\begin{aligned}
{\left[x, v_{2}\right]=} & {\left[x\left(r_{1}, t_{1}\right), x\left(r_{2}+r_{3}, u_{1}\right) x\left(r_{2}+r_{3}+r_{4}, u_{2}\right)\right] } \\
= & x\left(r_{1}+r_{2}+r_{3}+r_{4}, N\left(r_{1}, r_{2}+r_{3}+r_{4}\right) t_{1} u_{2}\right) \\
& \times x\left(r_{1}+r_{2}+r_{3}, N\left(r_{1}, r_{2}+r_{3}\right) t_{1} u_{1}\right)
\end{aligned}
$$

using Chevalley's commutator formula. Also

$$
\begin{aligned}
{\left[y, v_{1}\right]=} & x\left(r_{1}+r_{2}+r_{3}+r_{4}, N\left(r_{3}, r_{1}+r_{2}+r_{4}\right) a t_{1} d_{3}\right) \\
& \times x\left(r_{1}+r_{2}+r_{3}, N\left(r_{3}, r_{1}+r_{2}\right) a t_{1} d_{1}\right)
\end{aligned}
$$

again by Chevalley's formula.

Now $\left[y, v_{1}\right]^{v_{2}}=\left[y, v_{1}\right]$. Therefore

$$
\begin{aligned}
& {\left[x y, v_{1} v_{2}\right]=x\left(r_{1}+r_{2}+r_{3}, N\left(r_{1}, r_{2}+r_{3}\right) t_{1} u_{1}+N\left(r_{3}, r_{1}+r_{2}\right) a t_{1} d_{1}\right)} \\
& \quad \times x\left(r_{1}+r_{2}+r_{3}+r_{4}, N\left(r_{1}, r_{2}+r_{3}+r_{4}\right) t_{1} u_{2}+N\left(r_{3}, r_{1}+r_{2}+r_{4}\right) a t_{1} d_{3}\right) .
\end{aligned}
$$

Therefore $\left[x y, v_{1} v_{2}\right] \in X\left(r_{0}\right)$ implies that

$$
u_{1}=-N\left(r_{1}, r_{2}+r_{3}\right) N\left(r_{3}, r_{1}+r_{2}\right) a d_{1}
$$

and

$$
u_{2}=-N\left(r_{1}, r_{2}+r_{3}+r_{4}\right) N\left(r_{3}, r_{1}+r_{2}+r_{4}\right) a d_{3}
$$

since $N(r, s)= \pm 1$ in these cases. This completes the proof of Lemma 3.6.

LEMMA 3.7. Let $x=x\left(r_{1}, t_{1}\right), y=x\left(r_{3}, t_{3}\right) x\left(r_{4}, a t_{3}\right), t_{1}, t_{3}$ and $a \neq 0$. If $q$ is odd then $C_{V},(x y)=\left\langle x^{\prime}\left(r_{1}+r_{2}+r_{3}, d_{1}\right) x^{\prime}\left(r_{1}+r_{2}+r_{4}, d_{2}\right)\right.$

$$
\begin{aligned}
& x^{\prime}\left(r_{2}+r_{3}+r_{4},-\left(t_{3} t_{1}^{-1}\right) N\left(r_{1}, r_{2}+r_{3}+r_{4}\right) N\left(r_{3}, r_{1}+r_{2}+r_{4}\right)\left(d_{2}+a d_{1}\right)\right), \\
& X^{\prime}\left(r_{1}+r_{2}+r_{3}+r_{4}\right)\left|d_{i} \in F, i=1,2\right\rangle .
\end{aligned}
$$

If $q$ is even then

$$
\begin{aligned}
C_{V},(x y)= & \left\langle x^{\prime}\left(r_{1}+r_{2}, d_{1}\right) x^{\prime}\left(r_{2}+r_{3}, t_{3} t_{1}^{-1} d_{1}\right) x^{\prime}\left(r_{2}+r_{4}, a\left(t_{3} t_{1}^{-1}\right) d_{1}\right)\right. \\
& \times x^{\prime}\left(r_{2}+r_{3}+r_{4}, a t_{3}^{2} t_{1}^{-1} d_{1}\right), \\
& x^{\prime}\left(r_{1}+r_{2}+r_{3}, d_{2}\right) x^{\prime}\left(r_{1}+r_{2}+r_{4}, d_{3}\right) x^{\prime}\left(r_{2}+r_{3}+r_{4},\left(t_{3} t_{1}^{-1}\right)\left(d_{3}+a d_{2}\right)\right), \\
& X^{\prime}\left(r_{1}+r_{2}+r_{3}+r_{4}\right)\left|d_{i} \in F, i=1,2,3\right\rangle .
\end{aligned}
$$

Here it should be noted that we are choosing $N(\bar{r}, \bar{s})=N(r, s)$ where $\bar{r}_{1}=r_{1}$, $\bar{r}_{2}=r_{2}, \bar{r}_{3}=r_{4}$ and $\bar{r}_{4}=r_{3}$. That we can so choose follows from Steinberg (1959), Lemma 3.2.

ProOf. Let $v_{1} v_{2}$ be an element of the preimage of $C_{V}(x y)$ in $V$ where $v_{i} \in V_{i}$, $i=1,2$. As before $\left[y, v_{2}\right]=1$ and so $v_{2}=x\left(r_{2}+r_{3}, u_{1}\right) x\left(r_{2}+r_{4},-N\left(r_{4}, r_{2}+r_{3}\right)\right.$ $\left.\times N\left(r_{3}, r_{2}+r_{4}\right) a u_{1}\right) x\left(r_{2}+r_{3}+r_{4}, u_{2}\right)$. 
Since we are choosing $N(\bar{r}, \bar{s})=N(r, s)$ we get

and so

$$
N\left(r_{4}, r_{2}+r_{3}\right) N\left(r_{3}, r_{2}+r_{4}\right)=1
$$

$$
v_{2}=x\left(r_{2}+r_{3}, u_{1}\right) x\left(r_{2}+r_{4},-a u_{1}\right) x\left(r_{2}+r_{3}+r_{4}, u_{2}\right) .
$$

Computing we get

$$
\begin{aligned}
{\left[x, v_{2}\right]=} & x\left(r_{1}+r_{2}+r_{3}+r_{4}, N\left(r_{1}, r_{2}+r_{3}+r_{4}\right) t_{1} u_{2}\right) x\left(r_{1}+r_{2}+r_{4},\right. \\
& \left.N\left(r_{1}, r_{2}+r_{4}\right)\left(-a t_{1} u_{1}\right)\right) x\left(r_{1}+r_{2}+r_{3}, N\left(r_{1}, r_{2}+r_{3}\right) t_{1} u_{1}\right) z
\end{aligned}
$$

where $z \in Z(V)=X\left(r_{0}\right)$. In similar fashion

$$
\begin{aligned}
{\left[y, v_{1}\right]=} & x\left(r_{1}+r_{2}+r_{3}+r_{4}, N\left(r_{3}, r_{1}+r_{2}+r_{4}\right) t_{3} d_{3}\right. \\
& \left.+N\left(r_{3}, r_{1}+r_{2}\right) N\left(r_{1}+r_{2}+r_{3}, r_{4}\right) a t_{3}^{2} d_{1}+N\left(r_{4}, r_{1}+r_{2}+r_{3}\right) a t_{3} d_{2}\right) \\
& \times x\left(r_{1}+r_{2}+r_{3}, N\left(r_{3}, r_{1}+r_{2}\right) d_{1} t_{3}\right) x\left(r_{1}+r_{2}+r_{4}, N\left(r_{4}, r_{1}+r_{2}\right) d_{1} a t_{3}\right) .
\end{aligned}
$$

Now $\left[y, v_{1}\right]^{v_{2}}=\left[y, v_{1}\right] \bmod Z(V)$. Therefore

$$
\begin{aligned}
{\left[x y, v_{1} v_{2}\right] \bmod Z(V)=x\left(r_{1}+r_{2}+r_{3}, N\left(r_{1}, r_{2}+r_{3}\right) t_{1} u_{1}+N\left(r_{3}, r_{1}+r_{2}\right) d_{1} t_{3}\right) } \\
\quad \times x\left(r_{1}+r_{2}+r_{4}, N\left(r_{1}, r_{2}+r_{4}\right)\left(-a t_{1} u_{1}\right)+N\left(r_{4}, r_{1}+r_{2}\right) d_{1} a t_{3}\right) \\
\quad \times x\left(r_{1}+r_{2}+r_{3}+r_{4}, N\left(r_{1}, r_{2}+r_{3}+r_{4}\right) t_{1} u_{2}+N\left(r_{3}, r_{1}+t_{2}+r_{4}\right) t_{3} d_{3}\right. \\
\left.\quad+N\left(r_{4}, r_{1}+r_{2}+r_{3}\right) a t_{3} d_{2}+N\left(r_{3}, r_{1}+r_{2}\right) N\left(r_{1}+r_{2}+r_{3}, r_{4}\right) a t_{3}^{2} d_{1}\right) .
\end{aligned}
$$

Therefore $\left[x y, v_{1} v_{2}\right] \in Z(V)$ implies that

(a) $u_{1}=-N\left(r_{1}, r_{2}+r_{3}\right) N\left(r_{3}, r_{1}+r_{2}\right) t_{3} t_{1}^{-1} d_{1}$,

(b) $u_{1}=N\left(r_{1}, r_{2}+r_{4}\right) N\left(r_{4}, r_{1}+r_{2}\right) t_{3} t_{1}^{-1} d_{1}$ and

(c) $u_{2}=-N\left(r_{1}, r_{2}+r_{3}+r_{4}\right) t_{1}^{-1}\left(N\left(r_{3}, r_{1}+r_{2}+r_{4}\right) t_{3} d_{3}\right.$

$$
\left.+N\left(r_{4}, r_{1}+r_{2}+r_{3}\right) a t_{3} d_{2}+N\left(r_{3}, r_{1}+r_{2}\right) N\left(r_{1}+r_{2}+r_{3}, r_{4}\right) a t_{3}^{2} d_{1}\right) \text {. }
$$

If $q$ is odd then (a) and (b) force $d_{1}=u_{1}=0$ since $N\left(r_{1}, r_{2}+r_{3}\right)=N\left(r_{1}, r_{2}+r_{4}\right)$ and $N\left(r_{3}, r_{1}+r_{2}\right)=N\left(r_{4}, r_{1}+r_{2}\right)$. In this case

$$
u_{2}=-N\left(r_{1}, r_{2}+r_{3}+r_{4}\right) N\left(r_{3}, r_{1}+r_{2}+r_{4}\right) t_{3} t_{1}^{-1}\left(d_{3}+a d_{2}\right) \text {. }
$$

If $q$ is even, since $N(r, s)=1$ in all cases, (a) and (b) are the same equation, namely $u_{1}=t_{3} t_{1}^{-1} d_{1}$, and (c) becomes $u_{2}=t_{1}^{-1}\left(t_{3} d_{3}+a t_{3} d_{2}+a t_{3}^{2} d_{1}\right)$. This completes the proof of Lemma 3.7 .

Let $A \in \mathrm{Ab}(U)$. Then $|A| \geqslant q^{6}$ since $U$ contains abelian groups of this order. Also (A) $\varphi=A_{1} \leqslant X \times Y$ and $|A|=\left|A_{1}\right||A \cap V|$. Note that $A \cap V \geqslant Z(V)=X\left(r_{0}\right)$ by the maximality of $A$ since $X\left(r_{0}\right) \leqslant Z(U)$. Suppose $a_{1} \in A_{1}$ and $v \in A \cap V$. Then $a_{1} v_{1} \in A$ for some $v_{1} \in V$. Then

$$
1=\left[a_{1} v_{1}, v\right]=\left[a_{1}, v\right]^{v_{1}}\left[v_{1}, v\right]
$$


Therefore $\left[a_{1}, v\right] \in Z(V)=[V, V]$ and so $\left[A_{1}, A \cap V\right] \leqslant Z(V)$. It follows that

$$
(A \cap V)^{\prime} \leqslant C_{V^{\prime}}\left(A_{1}\right) \text {. }
$$

Now $C_{V} \cdot\left(A_{1}\right)$ is a vector space uver $F$ and so $\left|C_{V},\left(A_{1}\right)\right|=q^{f}$ for some integer $f \geqslant 0$.

LEMMA 3.8. Let $x \in X$ and $y, y_{1} \in Y$ with $x, y_{1} \neq 1$. Then $x y$ and $y_{1}$ cannot both be elements of $A_{1}$.

Proof. As observed

$$
\begin{aligned}
V /[V, V]=V^{\prime} & \cong V(A, 1, \lambda) \otimes W \text { as an } M \times N \text {-module } \\
& \cong W \otimes W \text { as an } N \text {-module. }
\end{aligned}
$$

Choose $e, f$ as in Lemma 3.3. Now $e \otimes u+f \otimes v \in C_{Y},(x y)$ if and only if $u \in \operatorname{ker}_{W}(y-1)^{2}$ and $v=-u(y-1) / a$, and $e \otimes u+f \otimes v \in C_{V},(y)$ if and only if $u, v \in C_{W}(y)$. Therefore $e \otimes u+f \otimes v \in C_{V^{\prime}}(x y) \cap C_{V^{\prime}}(y)$ implies that $u \in \operatorname{ker}(y-1)^{2} \cap C_{W}(y)$ and $v=-u(y-1) / a$. Therefore $x y$ and $y \in A_{1}$ implies $\operatorname{dim} C_{V}\left(A_{1}\right) \leqslant \operatorname{dim} C_{W}(y)$. Identifying $W$ with $\left\langle X(r) \mid r \geqslant r_{2}, r \neq r_{1}\right\rangle$ we see easily that $y \neq 1$ gives $\operatorname{dim} C_{w}(y)=2$. Therefore $\operatorname{dim}$ $C_{V}\left(A_{1}\right) \leqslant 2$. Since $(A \cap V)^{\prime} \leqslant C_{V},\left(A_{1}\right)$ it follows that $|A \cap V| \leqslant q^{3}$. Now $|A| \geqslant q^{6}$ and so $\left|A_{1}\right| \geqslant q^{3}$. Recall that $Y=X\left(r_{3}\right) \times X\left(r_{4}\right)$ and so $|Y|=q^{2}$. Therefore $A=X \times Y$.

Now the preimage in $V$ of $C_{V},(X \times Y)$ is $\left\langle X\left(r_{1}+r_{2}+r_{3}+r_{4}\right), X\left(r_{0}\right)\right\rangle$. Therefore $|A \cap V| \leqslant q^{2}$ and so $|A|=\left|A_{1}\right||A \cap V| \leqslant q^{5}$ which is a contradiction. With this contradiction the proof of Lemma 3.8 is completed.

LEMMA 3.9. If $q \geqslant 3, b=x\left(r_{1}, t_{1}\right) x\left(r_{3}, t_{2}\right) x\left(r_{4}, t_{3}\right), t_{1}, t_{2}, t_{3} \neq 0$ cannot be an element of $A_{1}$.

Proof. Suppose $b \in A_{1}$.

Observation (a). If $x\left(r_{1}, d_{1}\right) z\left(r_{3}, d_{2}\right) x\left(r_{4}, d_{3}\right) \in A_{1}$ where at least one of the $d_{t}$ is zero then all of the $d_{t}$ are zero or we get a contradiction by the symmetry of $r_{1}, r_{3}$ and $r_{4}$ and Lemma 3.8.

Observation (b). If $x\left(r_{1}, d_{1}\right) x\left(r_{3}, d_{2}\right) x\left(r_{4}, d_{3}\right) \in A_{1}$ such that $d_{1}=t_{1}$ then either $d_{2}=t_{2}$ and $d_{3}=t_{3}$ or we get a contradiction again by Lemma 3.8. By the symmetry of $r_{1}, r_{3}$ and $r_{4}$ we could replace the condition $d_{1}=t_{1}$ by $d_{2}=t_{2}$ or $d_{3}=t_{3}$. And so our conclusion now reads: if $d_{i}=t_{i}$ for any $i$ then $d_{i}=t_{i}$ for all $i, 1 \leqslant i \leqslant 3$.

Observations (a) and (b) force $\left|A_{1}\right| \leqslant q$ and force elements of $A_{1}-\{1\}$ to be of the form $x\left(r_{1}, d\right) x\left(r_{3}, t\right) x\left(r_{4}, u\right)$ where $d, t, u \neq 0$. If $\left|A_{1}\right|<q$, then since by Lemma 3.4 $|A \cap V| \leqslant q^{5}$ we get $|A|<q^{6}$ which is a contradiction. Hence $\left|A_{1}\right|=q$.

If $q$ is odd we are done since by Lemma 3.7 we get that $\left|C_{V^{\prime}}(b)\right|=q^{3}$ which gives $|A \cap V| \leqslant q^{4}$ and so $|A| \leqslant q^{5}$-a contradiction. Suppose $q$ is even and $q \geqslant 4$. Since $q \geqslant 3$, we can choose two distinct non-identity elements of $A_{1}, x_{1}$ and $x_{2}$ where $x_{1}=x\left(r_{1}, c_{1}\right) x\left(r_{3}, c_{2}\right) x\left(r_{4}, a c_{3}\right)$ and $x_{2}=x\left(r_{1}, b_{1}\right) x\left(r_{3}, b_{3}\right) x\left(r_{4}, a_{1} b_{3}\right)$. If $C_{V},\left(x_{1}\right) \neq$ 
$C_{V},\left(x_{2}\right)$ then $\left|C_{V},\left(A_{1}\right)\right| \leqslant q^{3}$ by Lemma 3.7 and so $|A \cap V| \leqslant q^{4}$ which gives the contradiction $|A| \leqslant q^{5}$. Hence $C_{V},\left(x_{1}\right)=C_{V},\left(x_{2}\right)$.

Using the notation of Lemma 3.7 this implies (i) $u_{1}=\left(c_{3} c_{1}^{-1}\right) d_{1}=\left(b_{3} b_{1}^{-1}\right) d_{1}$ for all $d_{1} \in F$ and (ii) $u_{2}=\left(c_{3} c_{1}^{-1}\right)\left(d_{3}+a d_{1} c_{3}+a d_{2}\right)=\left(b_{3} b_{1}^{-1}\right)\left(d_{3}+a_{1} d_{1} b_{3}+a_{1} d_{2}\right)$ for all $d_{1}, d_{2}, d_{3} \in F$. (i) implies $c_{3} c_{1}^{-1}=b_{3} b_{1}^{-1}$ which in conjunction with (ii) implies $a d_{1} c_{3}+a d_{2}=a_{1} d_{1} b_{3}+a_{1} d_{2}$ for all $d_{1}, d_{2} \in F$. This is a contradiction which we see as follows. If $a=a_{1}$ take $d_{1}=1$ and $d_{2}=0$ to get $c_{3}=b_{3}$ (recall $x_{1} \neq x_{2}$ implies $\left.c_{3} \neq b_{3}\right)$; if $a \neq a_{1}$ take $d_{1}=0$ and $d_{2}=1$ to get $a=a_{1}$. This completes the proof of Lemma 3.9.

LEMMA 3.10. If $|F|=2$ and $x=x\left(r_{1}, 1\right) x\left(r_{3}, 1\right) x\left(r_{4}, 1\right) \in A_{1}$ then the only possibility for $A$ is

where

$$
A^{*}=\left\{x v, V_{1} \mid v \in x\left(r_{2}+r_{3}+r_{4}, 1\right) V_{1}\right\}
$$

$$
\begin{aligned}
V_{1}=\langle & x\left(r_{1}+r_{2}, 1\right) x\left(r_{2}+r_{3}, 1\right) x\left(r_{2}+r_{4}, 1\right) x\left(r_{2}+r_{3}+r_{4}, 1\right), \\
& x\left(r_{1}+r_{2}+r_{3}, d_{1}\right) x\left(r_{1}+r_{2}+r_{4}, d_{2}\right) x\left(r_{2}+r_{3}+r_{4}, d_{1}+d_{2}\right), \\
& X\left(r_{1}+r_{2}+r_{3}+r_{4}\right), X\left(r_{0}\right)\left|d_{1}, d_{2} \in F\right\rangle .
\end{aligned}
$$

Proof. As in Lemma 3.9 $\left|A_{1}\right|=q=2$ in this case and so $A_{1}=\{1, x\}$. Let

and

$$
y_{1}=x\left(r_{1}+r_{2}, 1\right) x\left(r_{2}+r_{3}, 1\right) x\left(r_{2}+r_{4}, 1\right) x\left(r_{2}+r_{3}+r_{4}, 1\right)
$$

$$
y_{2}\left(d_{1}, d_{2}\right)=x\left(r_{1}+r_{2}+r_{3}, d_{1}\right) x\left(r_{1}+r_{2}+r_{4}, d_{2}\right) x\left(r_{2}+r_{3}+r_{4}, d_{1}+d_{2}\right) .
$$

Using Lemma 3.7 one gets that the preimage $V_{1}$ of $C_{V},\left(A_{1}\right)$ in $V$ equals

$$
\left\langle y_{1}, y_{2}\left(d_{1}, d_{2}\right), X\left(r_{1}+r_{2}+r_{3}+r_{4}\right), X\left(r_{0}\right) \mid d_{1}, d_{2} \in F\right\rangle \text {. }
$$

One checks that $V_{1}$ is abelian.

Now $\left|A_{1}\right|=2$ and $|A| \geqslant 2^{6}$ forces $|A \cap V| \geqslant 2^{5}$. But $A \cap V \leqslant V_{1}$ and so $A \cap V=V_{1}$. Now $\left[x, y_{1}\right]=x\left(r_{4}, 1\right),\left[x, y_{2}\left(d_{1}, d_{2}\right)\right]=1$ and $[x, W]=1$, where

$$
W=X\left(r_{1}+r_{2}+r_{3}+r_{4}\right) \times X\left(r_{0}\right) \text {. }
$$

Let $a \in A-(A \cap V)$. Then $a=x v_{1}$ for some $v_{1} \in V$. Now $[a, A \cap V]=1$ forces $\left[v_{1}, y_{1}\right]=$ $x\left(r_{0}, 1\right),\left[v_{1}, y_{2}\left(d_{1}, d_{2}\right)\right]=1$ and $\left[v_{1}, W\right]=1$. Hence $v_{1} \in C\left(\left\langle y_{2}\left(d_{1}, d_{2}\right), W \mid d_{1}, d_{2} \in F\right\rangle\right)$ which turns out to be $\left\langle V_{1}, x\left(r_{2}+r_{3}+r_{4}, 1\right)\right\rangle$. Now $\left[v_{1}, y_{1}\right]=x\left(r_{0}, 1\right)$ gives

$$
v_{1} \in x\left(r_{2}+r_{3}+r_{4}, 1\right) V_{1} \text {. }
$$

So we have that $A$ is a subset of $A^{*}$. One computes that $A^{*}$ is an abelian group of order $2^{6}$ and so $A=A^{*}$. This completes the proof of Lemma 3.10 .

Lemma 3.11. If $x\left(r_{1}, t_{1}\right) x\left(r_{3}, a t_{1}\right) \in A_{1}, t_{1} \neq 0$ then $A_{1}=\left\langle x\left(r_{1}, t\right) x\left(r_{3}, a t\right) \mid t \in F\right\rangle$.

Proof. (a) Suppose $a=0$. Then by Lemma 3.8 and the symmetry of $r_{1}, r_{3}$ and $r_{4}$ we get that $A_{1}$ contains no non-identity elements of the form $x\left(r_{3}, b_{1}\right) x\left(r_{4}, b_{2}\right)$ or 
$x\left(r_{1}, c_{1}\right) x\left(r_{4}, c_{2}\right), c_{2} \neq 0$, or $x\left(r_{1}, d_{1}\right) x\left(r_{3}, d_{2}\right) x\left(r_{4}, d_{3}\right), d_{i} \neq 0$, all $i$. (b) Suppose $a \neq 0$. Then $A_{1}$ contains no non-identity elements of the form

$$
x\left(r_{3}, b_{1}\right) x\left(r_{4}, b_{2}\right) \text { or } x\left(r_{1}, c_{1}\right) x\left(r_{4}, c_{2}\right) \text { or } x\left(r_{1}, d_{1}\right) x\left(r_{3}, d_{2}\right) x\left(r_{4}, d_{3}\right) \text {, }
$$

again by Lemma 3.8 and the symmetry of $r_{1}, r_{3}$ and $r_{4}$. (a) and (b) force $A_{1} \leqslant X\left(r_{1}\right) \times X\left(r_{3}\right)$ and so $\left|A_{1}\right| \leqslant q^{2}$. Note we know $\left|A_{1}\right| \geqslant q$ since $|A \cap V| \leqslant q^{5}$ and $|A| \geqslant q^{6}$.

Suppose $x_{1}=x\left(r_{1}, t_{1}\right) x\left(r_{3}, a t_{1}\right)$ and $x_{2}=x\left(r_{1}, d_{1}\right) x\left(r_{3}, a_{1} d_{1}\right)$ be non-identity elements of $A_{1}$ with $a \neq a_{1}$. Then Lemma 3.6 gives us that

$$
\operatorname{dim} C_{V} \cdot\left(A_{1}\right) \leqslant \operatorname{dim} C_{V},\left(\left\langle x_{1}, x_{2}\right\rangle\right) \leqslant 2 .
$$

Therefore $|A \cap V| \leqslant q^{3}$ and so $|A| \leqslant q^{5}$-a contradiction. Hence $a$ must equal $a_{1}$ and this combined with $\left|A_{1}\right| \geqslant q$ forces the desired conclusion. This completes the proof of Lemma 3.11 .

One notes that by the symmetry of $r_{1}, r_{3}$ and $r_{4}$ we might just as well consider elements of the form $x\left(r_{3}, t_{1}\right) x\left(r_{4}, a t_{1}\right)$ and $x\left(r_{4}, t_{1}\right) x\left(r_{1}, a t_{1}\right), t_{1} \neq 0$, in Lemma 3.11 and we would get the appropriate conclusion.

LEMMA 3.12. $A \in \mathrm{Ab}(U)$ implies $|A|=q^{6}$.

Proof. Lemmas 3.10 and 3.11 tell us that the possibilities for $A_{1}$ are

$$
\left\langle x\left(r_{1}, t\right) x\left(r_{3}, a_{1} t\right) \mid t \in F\right\rangle, \quad\left\langle x\left(r_{3}, t\right) x\left(r_{4}, a_{2} t\right) \mid t \in F\right\rangle, \quad\left\langle x\left(r_{4}, t\right) x\left(r_{1}, a_{3} t\right) \mid t \in F\right\rangle,
$$

where $a_{1}, a_{2}$ and $a_{3}$ range over $F$, and $\left\langle x\left(r_{1}, t\right) x\left(r_{3}, t\right) x\left(r_{4}, t\right) \mid \mathrm{t} \in F\right\rangle$, this last one being possible only when $|F|=2$. Now each of these possibilities has order $q$. Since $|A \cap V| \leqslant q^{5}$ it follows that $|A| \leqslant q^{6}$. Since we know $|A| \geqslant q^{6}$ we get that $|A|=q^{6}$. Hence Lemma 3.12 .

LEMMA 3.13. If $q$ is odd the possibilities

$$
\begin{gathered}
A_{1}=\left\langle x\left(r_{1}, t\right) x\left(r_{3}, a t\right) \mid t \in F\right\rangle \text { or }\left\langle x\left(r_{3}, t\right) x\left(r_{4}, a t\right) \mid t \in F\right\rangle \text { or } \\
\left\langle x\left(r_{4}, t\right) x\left(r_{1}, a t\right) \mid t \in F\right\rangle
\end{gathered}
$$

do not occur when $a \neq 0$.

Proof. Suppose $A_{1}=\left\langle x\left(r_{1}, t\right) x\left(r_{3}, a t\right) \mid t \in F\right\rangle$ for some $a \neq 0$. Lemma 3.6 tells us that

$$
\begin{aligned}
C_{V},\left(A_{1}\right)= & \\
& \left\langle x^{\prime}\left(r_{1}+r_{2}, d_{1}\right) x^{\prime}\left(r_{2}+r_{3},-N\left(r_{3}, r_{1}+r_{2}\right) N\left(r_{1}, r_{2}+r_{3}\right) a d_{1}\right),\right. \\
& x^{\prime}\left(r_{1}+r_{2}+r_{4}, d_{2}\right) x^{\prime}\left(r_{2}+r_{3}+r_{4},-N\left(r_{3}, r_{1}+r_{2}+r_{4}\right) N\left(r_{1}, r_{2}+r_{3}+r_{4}\right) a d_{2}\right), \\
& X^{\prime}\left(r_{1}+r_{2}+r_{3}\right), X^{\prime}\left(r_{1}+r_{2}+r_{3}+r_{4}\right)\left|d_{i} \in F\right\rangle .
\end{aligned}
$$


To simplify calculations choose $N(\bar{r}, \bar{s})=N(r, s)$ where now $\bar{r}_{1}=r_{3}, \bar{r}_{2}=r_{2}$, $\bar{r}_{3}=r_{1}$ and $r_{4}=\bar{r}_{4}$. Steinberg (1959), Lemma 3.2 again justifies the legitimacy of this procedure. Then

$$
\begin{aligned}
& C_{V},\left(A_{1}\right)=\left\langle x^{\prime}\left(r_{1}+r_{2}, d_{1}\right) x^{\prime}\left(r_{2}+r_{3},-a d_{1}\right), x^{\prime}\left(r_{1}+r_{2}+r_{4}, d_{2}\right) x^{\prime}\left(r_{2}+r_{3}+r_{4},-a d_{2}\right),\right. \\
& X^{\prime}\left(r_{1}+r_{2}+r_{3}\right), X^{\prime}\left(r_{1}+r_{2}+r_{3}+r_{4}\right)\left|d_{i} \in F\right\rangle .
\end{aligned}
$$

Now

Since

$$
\begin{aligned}
& {\left[x\left(r_{1}+r_{2}, d_{1}\right) x\left(r_{2}+r_{3},-a d_{1}\right), x\left(r_{1}+r_{2}+r_{4}, d_{2}\right) x\left(r_{2}+r_{3}+r_{4},-a d_{2}\right)\right]} \\
& \quad=\left[x\left(r_{1}+r_{2}, d_{1}\right), x\left(r_{2}+r_{3}+r_{4},-a d_{2}\right)\right]\left[x\left(r_{2}+_{3},-a d_{1}\right), x\left(r_{1}+r_{2}+r_{4}, d_{2}\right)\right] \\
& \quad=x\left(r_{0},\left[N\left(r_{1}+r_{2}, r_{2}+r_{3}+r_{4}\right)+N\left(r_{2}+r_{3}, r_{1}+r_{2}+r_{4}\right)\right]\left(-a d_{1} d_{2}\right)\right) .
\end{aligned}
$$

$$
N\left(r_{1}+r_{2}, r_{2}+r_{3}+r_{4}\right)=N\left(\overline{r_{1}+r_{2}}, \overline{r_{2}+r_{3}+r_{4}}\right)=N\left(r_{2}+r_{3}, r_{1}+r_{2}+r_{4}\right)
$$

these elements commute if and only if $q$ is even.

Therefore, if $q$ is odd, then $A \cap V$ is properly contained in the preimage of $C_{V},\left(A_{1}\right)$ in $V$. This means that $|A \cap V|<q^{5}$ and as a consequence $|A|<q^{6}$-a contradiction. Thus $A_{1}=\left\langle x\left(r_{1}, t\right) x\left(r_{3}, a t\right) \mid t \in F\right\rangle$ is not a possibility if $q$ is odd and $a \neq 0$. The two other configurations for $A_{1}$ are disposed of in like manner. This completes the proof of Lemma 3.13.

We are ready now to wind up the proof of Theorem 3.1. If $q=2$ and $A_{1}=$ $\left\langle x\left(r_{1}, t\right) x\left(r_{3}, t\right) x\left(r_{4}, t\right) \mid t \in F\right\rangle$ then by Lemma $3.10 A=A^{*}$. The other possibilities when $q$ is even are $A_{1}=\left\langle x\left(r_{1}, t\right) \times x\left(r_{3}, a t\right) \mid t \in F\right\rangle$ for some $a \in F$ and the $r_{3}-r_{4}$ and $r_{4}-r_{1}$ mixtures. Suppose $A_{1}=\left\langle x\left(r_{1}, t\right) x\left(r_{3}, a t\right) \mid t \in F\right\rangle$. One checks that the preimage $V_{1}$ of $C_{V},\left(A_{1}\right)$ in $V$ is abelian of order $q^{5}$. Now $A \cap V \leqslant V_{1}$ and in fact $A \cap V=V_{1}$ by order considerations. One has that $A \cap V \in \mathrm{Ab}(V),\left[A_{1}, A \cap V\right]=1$ and so we prove that $A=A_{1} \times(A \cap V)$ which turns out to be $B(1, a)$. The other remaining possibilities for $A_{1}$ are handled in similar fashion.

If $q$ is odd $A_{1}$ is one of $X\left(r_{1}\right), X\left(r_{3}\right)$ or $X\left(r_{4}\right)$. If $A_{1}=X\left(r_{1}\right)$ then $A=B_{1}$, if $A_{1}=X\left(r_{3}\right)$ then $A=B_{2}$ finally if $A=X\left(r_{4}\right)$ then $A=B_{3}$. This completes the proof of Theorem 3.1.

Proof of Theorem 3.2. We start with

Lemma 3.14. Let $y \in Y, y \neq 1$. Then $\operatorname{dim} C_{w}(y) \leqslant 2 n-6$.

Proof. Recall $W=V\left(D, n-2, \lambda_{1}\right)$ as an $N$-module. Then $N$ is represented on $W$ as a subgroup of the orthogonal group $O^{+}(2 n-4, q)$ using the notation of Carter (1972), p. 6, $Y$ being represented faithfully. Since $N=[N, N], N$ maps into (actually onto) $\Omega(2 n-4, q)=\left[O^{+}(2 n-4, q), O^{+}(2 n-4, q)\right]$. However, $\Omega(2 n-4, q)$ contains no transvections and hence our lemma follows immediately.

The proof is by induction on $n$. Let $A \in \mathrm{Ab}(U)$. First we consider the case $n=5$. 
Then $|A| \geqslant q^{10}$ since abelian subgroups of $U$ of this order exist. As usual $(A) \varphi=$ $A_{1} \leqslant X \times Y$ and $|A|=\left|A_{1}\right||A \cap V|$. Now $N \cong A_{3}(q)$ and so by Theorem 2.1(a) $Y$ has a unique largest abelian subgroup $\left\langle X(r) \mid r \geqslant r_{3}, r \neq r_{2}\right\rangle$ of order $q^{4}$. Hence $\left|A_{1}\right| \leqslant q^{5}$. Now by Lemma 3.4 we have $|A \cap V| \leqslant q^{7}$. Hence $|A| \leqslant q^{12}$.

Lemma 3.15. Let $x \in X, y \in Y$. Then no element of the form $x y$ occurs in $A_{1}$ where $x \neq 1$. Hence $A_{1} \leqslant Y$.

Proof. Suppose there exists $x y \in A_{1}$ with $x \neq 1$. Note that $\left|A_{1}\right| \geqslant q^{3}$ since $|A \cap V| \leqslant q^{7}$. We claim that there exists $y_{1} \in Y \cap A$ with $y_{1} \neq 1$. Suppose not. Then all elements of $A_{1}$ are of the form $x_{2} y_{2}$ where $x_{2}=1$ implies $y_{2}=1$. If there exists $x_{1} \in X$ such that $x_{1} y_{3}$ and $x_{1} y_{4} \in A_{1}$ with $y_{3} \neq y_{4}$ then $y_{1}=y_{3} y_{4}^{-1} \in A_{1}$ which is a contradiction. If there exists no such $x_{1}$ then $\left|A_{1}\right| \leqslant q$ which is another contradiction. Our claim holds.

So $x y$ and $y_{1} \in A_{1}$. Now $C_{V},\left(A_{1}\right) \leqslant C_{V},(x y) \cap C_{V},\left(y_{1}\right)$. Choose $\{e, f\}$ as in Lemma 3.3. Then $e \otimes u+f \otimes v \in C_{V},(x y) \cap C_{V},\left(y_{1}\right)$ implies $u \in \operatorname{ker}_{W}(y-1)^{2} \cap C_{W}\left(y_{1}\right)$ and $v=$ $-u(y-1) / a$. Hence $\operatorname{dim} C_{V}\left(A_{1}\right) \leqslant \operatorname{dim} C_{W}\left(y_{1}\right) \leqslant 4$ by Lemma 3.14. Therefore $|A \cap V| \leqslant q^{5}$. This forces $\left|A_{1}\right| \geqslant q^{5}$ which in turn forces $A_{1}$ to be the unique largest abelian subgroup of $X \times Y$ of order $q^{5}$, namely $X \times\left\langle X(r) \mid r \geqslant r_{3}, r \neq r_{2}\right\rangle$. Easy calculation gives $C_{V},\left(A_{1}\right)=X^{\prime}\left(r_{1}+r_{2}+r_{3}+r_{4}\right)$. Hence $|A \cap V| \leqslant q^{2}$ and $|A| \leqslant q^{7}$-a contradiction. This completes the proof of Lemma 3.15.

Lemma 3.16. $\left|A_{1}\right|=q_{3}$. If $q$ is even then

$$
A_{1}=\left\langle x\left(r_{4}, t\right) x\left(r_{5}, a t\right), x\left(r_{3}+r_{4}, d\right) x\left(r_{3}+r_{5}, a d\right), X\left(r_{3}+r_{4}+r_{5}\right) \mid t, d \in F\right\rangle
$$

for some fixed $a \in F$ or $A=\left\langle X\left(r_{5}\right), X\left(r_{3}+r_{5}\right), X\left(r_{3}+r_{4}+r_{5}\right)\right\rangle$. If $q$ is odd then $A_{1}$ is one of the two choices which remain when $a=0$.

Proof. $A_{1} \leqslant Y$ by Theorem 3.15. This implies $\left|A_{1}\right| \leqslant q^{4}$ since $Y$ has a unique largest abelian subgroup of order $q^{4}$.

Suppose $\operatorname{dim} C_{W}\left(A_{1}\right) \leqslant 2$. Then $\operatorname{dim} C_{V^{\prime}}\left(A_{1}\right)=2 \operatorname{dim} C_{W}\left(A_{1}\right) \leqslant 4$ which gives $|A \cap V| \leqslant q^{6}$ and $|A| \leqslant q^{9}$-a contradiction. Hence $\operatorname{dim} C_{W}(A) \geqslant 3$. Identifying $W$ with $\left\langle X(r) \mid r \geqslant r_{2}, r \neq r_{1}\right\rangle$ we get that $A_{1} \times C_{W}\left(A_{1}\right)$ is an abelian subgroup contained in $P=\left\langle X(r) \mid r \in \Phi_{L}^{+}\right\rangle$where $L=\Pi-\left\{r_{1}\right\}$. Now $P \in \operatorname{Syl}_{p}\left(G_{1}\right)$ where $G_{1}=\left\langle X(r) \mid r \in \Phi_{L}\right\rangle \cong$ $D_{4}(q)$. Hence Theorem 3.1 implies $\left|A_{1} \times C_{W}\left(A_{1}\right)\right| \leqslant q^{6}$.

Therefore $\left|A_{1}\right| \leqslant q^{3}$. But we know already that $\left|A_{1}\right| \geqslant q^{3}$. Hence $\left|A_{1}\right|=q^{3}$ and $\left|A_{1} \times C_{W}\left(A_{1}\right)\right|=q^{6}$, that is,$A_{1} \times C_{W}\left(A_{1}\right) \in \mathrm{Ab}(P)$. Checking through the elements of $\mathrm{Ab}(P)$ we find that $A_{1}$ is as desired. This concludes the proof of Lemma 3.16.

For all $A_{1}$ in Lemma 3.16 we find that the preimage $D_{1}$ of $C_{V},\left(A_{1}\right)$ in $V$ is an element of $\mathrm{Ab}(V)$ with $\left[A_{1}, D_{1}\right]=1$. Order considerations force $A \cap V=D_{1}$ and we prove 
that $A=A_{1} D_{1}$ in our usual fashion. This settles Theorem 3.2 for $n=5$. Assume now that Theorem 3.2 is true for any integer $k$ such that $5 \leqslant k<n$ and we deal with $U \in \operatorname{Syl}_{p} D_{n}(q)$. Let $A \in \mathrm{Ab}(U)$. Then $|A| \geqslant q^{n(n-1)} / 2$ since abelian groups of this order exist in $U$. As usual $(A) \varphi=A_{1} \leqslant X \times Y$ and $|A|=\left|A_{1}\right||A \cap V|$. By Lemma 3.4 $|A \cap V| \leqslant q^{2 n-3}$. If $L \in \mathrm{Ab}(Y)$ then $|L|=q^{(n-2)(n-3) / 2}$ by our induction hypothesis and Theorem 3.1. Therefore $\left|A_{1}\right| \leqslant q^{(n-2)(n-3) / 2+1}$ and $|A| \leqslant q^{(n-2)(n-3) / 2+2 n-2}=$ $q^{n(n-1) / 2+1}$.

Lemma 3.17. Let $x \in X, y \in Y$. Then no element of the form $x y$ occurs in $A_{1}$ where $x \neq 1$. Hence $A_{1} \leqslant Y$.

Proof. Suppose $x y \in A_{1}$ where $x \neq 1$. Exactly the same argument as was used in Lemma 3.15 forces $|A \cap V| \leqslant q^{2 n-5}$. Hence $|A| \leqslant q^{(n-2)(n-3) / 2+2 n-4}=q^{n(n-1) / 2-1}-\mathrm{a}$ contradiction. Hence Lemma 3.17.

Lemma 3. 18. $A_{1} \in \mathrm{Ab}(Y)$. If $q$ is odd then $A_{1}$ is one of $C_{1}, C_{2}$ where $C_{1}=\langle X(r)| r \geqslant$ $\left.r_{n-1}, r \neq r_{2}\right\rangle$ and $C_{2}=\left\langle X(r) \mid r \geqslant r_{n}, r \neq r_{2}\right\rangle$. If $q$ is even then $A_{1}=C_{2}$ or $A=C(1, a)$ for some $a \in F$ where

$$
\begin{array}{r}
C(1, a)=\langle x(r, t) x(\bar{r}, a t), X(s)| r \geqslant r_{n-1}, r \neq r_{n-2}+r_{n-1}+r_{n}, s \geqslant r_{n-2}+r_{n-1}+r_{n}, \\
\left.r, s \neq r_{2}, t \in F\right\rangle .
\end{array}
$$

Proof. Lemma 3.17 implies $A_{1} \leqslant Y$ and so induction with Theorem 3.1 forces $\left|A_{1}\right| \leqslant q^{(n-2)(n-3) / 2}$. Order considerations force equality and so $A_{1} \in \mathrm{Ab}(Y)$. Induction again with Theorem 3.1 gives us $\mathrm{Ab}(Y)$. The list is not exclusive enough for our purposes and we eliminate the undesirable options by checking that in each case $\operatorname{dim} C_{W}\left(A_{1}\right)<n-2$. This implies that $\left|C_{V^{\prime}}\left(A_{1}\right)\right|<q^{2 n-4}$. Since $C_{V^{\prime}}\left(A_{1}\right)$ is an $F$-vector space $\left|C_{V},\left(A_{1}\right)\right| \leqslant q^{2 n-5}$. Hence $|A \cap V| \leqslant q^{2 n-4}$ and $|A| \leqslant q^{(n-2)(n-3) / 2+2 n-4}=$ $q^{n(n-1) / 2-1}-$ a contradiction. This completes the proof of Lemma 3.18.

For all $A_{1}$ in the conclusion of Lemma 3.18 the preimage $D_{1}$ of $C_{V},\left(A_{1}\right)$ in $V$ is an element of $\mathrm{Ab}(V)$ with $\left[A_{1}, D_{1}\right]=1$. Order considerations force $A \cap V=D_{1}$ and we prove that $A=A_{1} D_{1}$ in our usual fashion. For $A_{1}=C_{2}$ then $A=B_{2}$ and for $A_{1}=C(1, a)$ then $A=B(1, a)$. This completes the proof of Theorem 3.2.

\section{The solution for $B_{n}(q), q$ even}

With this section the lacuna in Section 2 can be filled thanks to the fact that $B_{n}(q) \cong C_{n}(q)$ if $q$ is even. Let $q=2^{m}, m \geqslant 1$.

THEOREM 4.1. Let $G=B_{2}(q)$. Let

$$
\begin{aligned}
& A_{1}=\left\langle X\left(r_{1}\right), X\left(r_{1}+r_{2}\right), X\left(r_{1}+2 r_{2}\right)\right\rangle, \\
& A_{2}=\left\langle X\left(r_{2}\right), X\left(r_{1}+r_{2}\right), X\left(r_{1}+2 r_{2}\right)\right\rangle, \\
& A_{3}=\left\langle x\left(r_{1}, t\right) x\left(r_{2}, t\right), X\left(r_{1}+r_{2}\right), X\left(r_{1}+2 r_{2}\right)\right\rangle .
\end{aligned}
$$




\section{Then}

(a) if $q=2, \mathrm{Ab}(U)=\left\{A_{1}, A_{2}, A_{3}\right\}$,

(b) if $q>2, \mathrm{Ab}(U)=\left\{A_{1}, A_{2}\right\}$.

THEOREM. 4.2. Let $G=B_{n}(q)$ with $n \geqslant 3$ and let $B=\left\langle X(r) \mid r \geqslant r_{n}\right\rangle$. Then $\operatorname{Ab}(U)=$ $\{B\}$ and $|B|=q^{n(n+1) / 2}$.

COROLlaRY 4.3. Let $G=C_{n}(q)$ with $n \geqslant 3$ and let $B=\left\langle X(r) \mid r \geqslant r_{n}\right\rangle$. Then $\mathrm{Ab}(U)=$ $\{B\}$ and $|B|=q^{n(n+1) / 2}$.

Proof of Theorem. 4.1. Since $q$ is even $Z(U)=\left\langle X\left(r_{1}+r_{2}\right), X\left(r_{1}+2 r_{2}\right)\right\rangle$. Therefore $A \in \mathrm{Ab}(U)$ implies that $Z(U) \leqslant A$. It follows that in order to pin down the elements of $\mathrm{Ab}(U)$ we need only inspect $C_{U}(x)$ where $x=x\left(r_{1}, d_{1}\right), x\left(r_{2}, d_{2}\right)$ or $x\left(r_{1}, d_{1}\right) x\left(r_{2}, d_{2}\right)$, $d_{1}, d_{2} \neq 0$. Now $C_{U}\left(x\left(r_{1}, d_{1}\right)\right)=A_{1}$ and $C_{U}\left(x\left(r_{2}, d_{2}\right)\right)=A_{2}$.

Finally let $x\left(r_{1}, e_{1}\right) x\left(r_{2}, e_{2}\right) \in C_{U}\left(x\left(r_{1}, d_{1}\right) x\left(r_{2}, d_{2}\right)\right)$. Then

$$
\begin{aligned}
1 & =\left[x\left(r_{1}, d_{1}\right) x\left(r_{2}, d_{2}\right), x\left(r_{1}, e_{1}\right) x\left(r_{2}, e_{2}\right)\right] \\
& =x\left(r_{1}+r_{2}, d_{1} e_{2}+d_{2} e_{1}\right) x\left(r_{1}+2 r_{2}, d_{1} e_{2}^{2}+e_{1} d_{2}^{2}\right)
\end{aligned}
$$

if and only if $d_{1} e_{2}=d_{2} e_{1}$ and $d_{1} e_{2}^{2}=e_{1} d_{2}^{2}$

if and only if $e_{1}=d_{1} d_{2}^{-1} e_{2}$ and $e_{2}^{2}=e_{2} d_{2}$,

if and only if $e_{1}=d_{1}$ and $e_{2}=d_{2}$ or $e_{1}=e_{2}=0$.

Gathering together these pieces of information on centralizers we have Theorem 4.1.

ProOf of TheOREM. 4.2. The proof will be by induction on $n$. First we set up some notation. Let $P_{J}$ be the parabolic subgroup of $G$ associated with $J=\Pi-\left\{r_{2}\right\}$. Then, if necessary, by Barry (1977), Theorem 3.13 we have $S_{J}=M \times N$ where $M=$ $\left\langle X\left(r_{1}\right), X\left(-r_{1}\right)\right\rangle \cong A_{1}(q)$ and $N=\left\langle X(r) \mid r \in \Phi_{K}\right\rangle \cong B_{n-2}(q)$ where $K=\Pi-\left\{r_{1}, r_{2}\right\}$.

Now $U_{J}=\left\langle X(r) \mid r \geqslant r_{2}\right\rangle$ and $\left[U_{J}, U_{J}\right]=X\left(r_{0}\right)$ where $r_{0}=r_{1}+2 r_{2}+\ldots+2 r_{n}$ is the highest root. However, since $q$ is even

$$
Z\left(U_{J}\right)=\left\langle X\left(r_{2}+r_{3}+\ldots+r_{n}\right), X\left(r_{1}+r_{2}+\ldots+r_{n}\right), X\left(r_{0}\right)\right\rangle .
$$

Let $V=U_{J}$ from now on. $M \times N$ acts on $V$ by right conjugation and by Barry (1977), Theorem 3.13 we have

$$
\begin{aligned}
V /[V, V] & \cong V\left(A, 1, \lambda_{1}\right) \otimes W \text { as an } M \times N \text {-module } \\
& \cong W \otimes W \text { as an } N \text {-module, }
\end{aligned}
$$

where $W=V\left(B, n-2, \lambda_{1}\right)$ can be identified with $\left\langle X(r) \mid r \geqslant r_{2}, r \neq r_{1}\right\rangle$.

Let $X=X\left(r_{1}\right)$ and $Y=\left\langle X(r) \mid r \in \Phi_{K}^{+}\right\rangle$. Then $X \times Y \in \operatorname{Syl}_{2}(M \times N)$ and $U=X Y V$. Let $\varphi$ be the natural epimorphism of $U$ on $U / V$ and ' that of $V$ on $V /[V, V]$.

Lemma 4.4. Let $C \in \mathrm{Ab}(V)$. Then $|C|=q^{2 n-1}$. 
Proof. Firstly we observe that $Z(V) \leqslant C$. Now $\bar{V}=V / Z(V)$ can be made into a vector space over $F$ and indeed $\bar{V}$ can be equipped with a non-degenerate alternating bilinear form ( , ) as follows:

$$
x\left(r_{0},\left(\bar{v}_{1}, \bar{v}_{2}\right)\right)=\left[v_{1}, v_{2}\right] \text { where } \bar{v}_{1}, \bar{v}_{2} \in \bar{V} .
$$

The non-degeneracy is assured by our factoring out by $Z(V)$.

Now it is clear, that for $W$ a subgroup of $V$, that $W$ is abelain if and only if $W$ is contained in a totally isotropic subspace of $\vec{V}$. The maximum dimension of such a subspace is $2 n-4$ since $|\bar{V}|=q^{4 n-8}$. Therefore $W$ abelian implies $|W| \leqslant q^{2 n-4}$ and so $|W| \leqslant q^{2 n-1}$. In fact abelian subgroups of $V$ of this order exist, for example, $\left\langle X(r) \mid r \geqslant r_{2}+\ldots+r_{n}\right\rangle$. Hence Lemma 4.4 holds.

One notes that Lemma 3.3 applies in the present context even though $Y, W, V /$ $[V, V]$ et cetera have changed meaning.

Let $A \in \mathrm{Ab}(U)$. Then $|A| \geqslant q^{6}=|B|$. Also $(A) \varphi=A_{1} \leqslant X \times Y=X\left(r_{1}\right) \times X\left(r_{3}\right)$ and $|A|=\left|A_{1}\right||A \cap V|$. Suppose $x y \in A_{1}$ with $x \in X, y \in Y$ and $x \neq 1$. Then by Lemma 3.3 we get $\operatorname{dim} C_{V},(x y) \leqslant \operatorname{dim} W=3$. Therefore $|A \cap V| \leqslant q^{4}$. Now $|A| \geqslant q^{6}$ forces $\left|A_{1}\right|=$ $q^{2}$ and hence $A_{1}=X \times Y$. But then $C_{V}\left(A_{1}\right)=\left\langle X^{\prime}\left(r_{1}+r_{2}+r_{3}\right), X^{\prime}\left(r_{1}+r_{2}+2 r_{3}\right)\right\rangle$. This implies that $|A \cap V| \leqslant q^{3}$ and $|A| \leqslant q^{5}$ which is a contradiction. Therefore $A_{1} \leqslant Y$ and order arguments force equality. The preimage $V_{1}=\left\langle X(r) \mid r \geqslant r_{2}+r_{3}\right\rangle$ of $C_{V},\left(A_{1}\right)$ in $V$ is an element of $\mathrm{Ab}(V)$. As in previous sections we prove $A=V_{1} Y=B$ to conclude the case of $n=3$.

Assume now that Theorem 4.2 is true for any integer $k$ such that $3 \leqslant k \leqslant n$ and we deal with $U \in \operatorname{Syl}_{2}\left(B_{n}(q)\right)$. Let $A \in \mathrm{Ab}(U)$. Then $|A| \geqslant|B|=q^{n(n+) 1 / 2}$ and $(A) \varphi=$ $A_{1} \leqslant X \times Y$. By induction $Y$ has a bound $q^{(n-1)(n-2) / 2}$ on abelian subgroups and so $\left|A_{1}\right| \leqslant q^{(n-1(n-2) / 2+1}$.

Suppose $x y \in A_{1}$, with $x \in X, y \in Y$ and $x \neq 1$. Then by Lemma 3.3, $\operatorname{dim} C_{V},(x y) \leqslant$ $\operatorname{dim} W=2 n-3$ and so $|A \cap V| \leqslant q^{2 n-2}$. Now

$$
q^{n(n+1) / 2} \leqslant|A|=\left|A_{1}\right| A \cap V \mid \leqslant q^{(n-1)(n-2) / 2+1} \cdot q^{2 n-2}=q^{n(n+1) / 2}
$$

implies $\left|A_{1}\right|=q^{(n-2)(n-1) / 2+1}$, which in turn implies $A_{1} \in \mathrm{Ab}(X \times Y)$ and so $A_{1}=$ $X \times E$ where $E \in \mathrm{Ab}(Y)$. Induction and Theorem 4.1 provide the possibilities for $E$ and by an easy calculation one finds that in each case $\left|C_{V},\left(A_{1}\right)\right| \leqslant q^{n-1}$. This gives $|A \cap V| \leqslant q^{n}$ and so $|A| \leqslant q^{(n-1)(n-2) / 2+1}, q^{n}<q^{n(n+1) / 2}$ since $n \geqslant 3$. We have arrived at a contradiction and so $A_{1} \leqslant Y$.

An order calculation forces $A_{1} \in \mathrm{Ab}(U)$. Again by induction and Theorem 4.1 we have $A_{1}=\left\langle X(r) \mid r \in \Phi_{K}^{+}, r \geqslant r_{n}\right\rangle$ if $\mathrm{n} \geqslant 5, A_{1}$ is one of $E_{i}, i=1,2,3$ if $n=4$ and $q=2$, while $A_{1}$ is one of $E_{i}, i=1,2$, if $n=4$ and $q>2$ where

and

$$
\begin{aligned}
& E_{1}=\left\langle X\left(r_{4}\right), X\left(r_{3}+r_{4}\right), X\left(r_{3}+2 r_{4}\right)\right\rangle, \\
& E_{2}=\left\langle X\left(r_{3}\right), X\left(r_{3}+r_{4}\right), X\left(r_{3}+2 r_{4}\right)\right\rangle
\end{aligned}
$$

$$
E_{3}=\left\langle x\left(r_{3}, t\right) x\left(r_{4}, t\right), X\left(r_{3}+r_{4}\right), X\left(r_{3}+2 r_{4}\right) \mid t \in F\right\rangle .
$$


One checks that $\left|C_{V},\left(E_{i}\right)\right| \leqslant q^{4}$ if $n=4$ and $i=2$ or 3 . Hence $|A \cap V| \leqslant q^{5}$ and $|A| \leqslant q^{8}$ which is a contradiction. In all cases then $A_{1}=\left\langle X(r) \mid r \in \Phi_{K}^{+}, r \geqslant r_{n}\right\rangle$. The preimage $V_{1}$ in $V$ of $C_{V},\left(A_{1}\right)=\left\langle X(r) \mid r \geqslant r_{2}+r_{3}+\ldots+r_{n}\right\rangle$ is an element of $\mathrm{Ab}(U)$. In our usual fashion then we get $A=A_{1} V_{1}=B$. This completes the proof of Theorem 4.2.

Proof of Corollary 4.3. Since $q$ is even, $C_{n}(q) \cong B_{n}(q)$ and so a 2-Sylow subgroup of $C_{n}(q)$ is isomorphic to a 2-Sylow subgroup of $B_{n}(q)$. If $n \geqslant 3$ Theorem 4.2 guarantees then a 2-Sylow subgroup of $B_{n}(q)$ and hence of $C_{n}(q)$ has a unique abelian subgroup of largest order $q^{n(n+1) / 2}$. Now $B=\left\langle X(r) \mid r \geqslant r_{n}\right\rangle$ is an abelian subgroup of order $q^{n(n+1) / 2}$ in the 2-Sylow subgroup $U$ of $C_{n}(q)$. Hence $\operatorname{Ab}(U)=\{B\}$.

\section{The solution for $B_{n}(q), q$ odd and $n \geqq 3$}

Let $q=p^{m}, p$ an odd prime and $m \geqslant 1$. Before we state the main results of this section we need to define some abelian subgroups of $U$. Firstly let $B=\left\langle X(r) \mid r \geqslant r_{1}\right\rangle$.

Next for $\left(a_{1}, a_{2}, \ldots, a_{n}\right) \in F^{n}-\{0\}$ we define

$$
\begin{array}{r}
B\left(a_{1}, a_{2}, \ldots, a_{n}\right)=\left\langle x\left(r_{n}, a_{1} t\right) x\left(r_{n-1}+r_{n}, a_{2} t\right) \ldots x\left(r_{1}+r_{2}+\ldots+r_{n}, a_{n} t\right),\right. \\
X(s)\left|s \geqslant r_{n-1}+2 r_{n}, t \in F\right\rangle .
\end{array}
$$

For $\left(a_{1}, a_{2}, \ldots, a_{n-1}\right) \in F^{n-1}-\{0\}$ we define

$$
\begin{aligned}
C\left(a_{1}, a_{2}, \ldots, a_{n-1}\right)= & \left\langle x\left(r_{n-1}+r_{n}, a_{1} t f\right) x\left(r_{n-2}+r_{n-1}+r_{n}, a_{2} t\right) \ldots\right. \\
& \times x\left(r_{1}+r_{2}+\ldots+r_{n}, a_{n-1} t\right), X(r), X(s) \mid r \geqslant r_{n-1}, \\
& \left.r \neq r_{n}, s \geqslant r_{n-2}+2 r_{n-1}+2 r_{n}, t \in F\right\rangle .
\end{aligned}
$$

Note that $B\left(a_{1}, a_{2}, \ldots, a_{n}\right)=B\left(b_{1}, b_{2}, \ldots, b_{n}\right)$ if and only if there exists $u \in F^{*}$ with $\left(a_{1}, a_{2}, \ldots, a_{n}\right)=u\left(b_{1}, b_{2}, \ldots, b_{n}\right)$. A similar remark holds for the groups $C\left(a_{1}, a_{2}\right.$, $\left.\ldots, a_{n-1}\right)$.

THEOREM 5.1. Let $G=B_{3}(q)$. Then

(a) $A \mathrm{~b}(U)=\{B\}$ and $|B|=q^{5}$,

(b) if $A$ is an abelian subgroup of $U$ not contained in $B$, then $|A| \leqslant q^{4}$.

THEOREM 5.2. Let $G=B_{4}(q)$. Then

$$
\begin{aligned}
\mathrm{Ab}(U)=\left\{B, B\left(a_{1}, a_{2}, a_{3}, a_{4}\right), C\left(b_{1}, b_{2}, b_{3}\right)^{x\left(r_{4}, t\right)} \mid\right. \\
\left.\left(a_{1}, a_{2}, a_{3}, a_{4}\right) \neq(0,0,0,0),\left(b_{1}, b_{2}, b_{3}\right) \neq(0,0,0), t \in F\right\} .
\end{aligned}
$$

Hence any element of $\mathrm{Ab}(U)$ has order $q^{7}$.

THEOREM 5.3. Let $G=B_{n}(q)$ where $n \geqslant 5$. Then $\operatorname{Ab}(U)=\left\{B\left(a_{1}, a_{2}, \ldots, a_{n}\right)\right.$, $\left.C\left(b_{1}, b_{2}, \ldots, b_{n-1}\right)^{x\left(r_{n}, t\right)} \mid\left(a_{1}, a_{2}, \ldots, a_{n}\right) \in F^{n}-\{0\},\left(b_{1}, b_{2}, \ldots, b_{n-1}\right) \in F^{n-1}-\{0\}, t \in F\right\}$. Hence any element of $\mathrm{Ab}(U)$ has order $q^{n(n-1) / 2+1}$. 
The notation we set up in Section 4 at the beginning of the proof of Theorem 2.4 will apply in this section also. However here $[V, V]=Z(V)=X\left(r_{0}\right)$ since we are in odd characteristic. As a consequence of this we record

LeMmA 5.4. If $C \in \mathrm{Ab}(V)$, then $|C|=q^{2 n-2}$.

LeMmA 5.5. If $y \in Y, y \neq 1$, then $\operatorname{dim} C_{W}(y) \leqslant \operatorname{dim} W-2=2 n-1$.

Proof. This is exactly the same as the proof of Lemma 3.14. One notes that Lemma 3.3 applies to this section as it did to section 4 .

Proof of Theorem 5.1. (a) Here $Y=X\left(r_{3}\right)$. Let $A \in \mathrm{Ab}(U)$. Then $|A| \geqslant|B|=q^{5}$. Since by Lemma $5.4|A \cap V| \leqslant q^{4}$ we get that $\left|A_{1}\right| \geqslant q^{2}$ where $A_{1}=(A) \varphi$. Suppose $x y \in A_{1}$ where $x \in X, y \in Y$ and $x, y \neq 1$. Then by Lemma 3.3

$$
\operatorname{dim} C_{V},(x y)=\operatorname{dim} \operatorname{ker}_{W}(y-1)^{2} .
$$

Now $y=x\left(r_{3}, t\right), t \neq 0$, and $\operatorname{ker}_{W}(y-1)^{2} \neq W$ since $x\left(r_{2}, 1\right)(y-1)^{2} \neq 0$.

$$
\begin{aligned}
\left(\left[\left[x\left(r_{2}, 1\right), x\left(r_{3}, t\right)\right], x\left(r_{3}, t\right)\right]=\left[x\left(r_{2}+r_{3}, \pm t\right) x\left(r_{2}+2 r_{3}, \pm t^{2}\right),\right.\right. \\
\left.x\left(r_{3}, t\right)\right]=x\left(r_{2}+2 r_{3}, \pm 2 t^{2}\right) \neq 1
\end{aligned}
$$

since $q$ is odd. The signs here depend only on structure constants). Therefore $\operatorname{dim} \operatorname{ker}_{W}(y-1)^{2} \leqslant 2$. It follows that $|A \cap V| \leqslant q^{3} .|A| \geqslant q^{5}$ implies $A_{1}=X \times Y$. But $C_{V},(X \times Y)=X^{\prime}\left(r_{1}+r_{2}+2 r_{3}\right)$ giving $|A \cap V| \leqslant q^{2}$ and $|A| \leqslant q^{4}$-a contradiction. Therefore $A_{1} \leqslant X$ or $A_{1} \leqslant Y$. Suppose that $A_{1} \leqslant Y$ and let $y \in Y, y \neq 1$. Then $C_{W}(y)=$ $X\left(r_{2}+2 r_{3}\right)$ and so $\operatorname{dim} C_{V},(y)=2 \operatorname{dim} C_{W}(y)=2$. Hence $|A \cap V| \leqslant q^{3}$ and $|A| \leqslant q^{4}-\mathrm{a}$ contradiction. Hence $A_{1} \leqslant X$ and now $\left|A_{1}\right| \geqslant q$ forces $A_{1}=X$. We get $A=B$ in our usual fashion.

(b) Suppose $A$ is an abelian subgroup of $U$ such that $|A|>q^{4}$ which is not contained in $B$. Then by Lemma $5.4 A \leqslant V$. Therefore $(A) \varphi=A_{1} \neq 1$. If $A_{1} \leqslant Y$ then $\left|A_{1}\right| \leqslant q$ and $|A \cap V| \leqslant q_{3}$ as in part (a) giving the contradiction $|A| \leqslant q^{4}$.

Suppose now that $a=x\left(r_{1}, t_{1}\right) x\left(r_{3}, t_{3}\right) \in A_{1}, t_{1}, t_{3} \neq 0$. Then $\operatorname{dim} C_{V},(a)=2$ since dim $\operatorname{ker}_{W}\left(x\left(r_{3}, t_{3}\right)-1\right)^{2}=2$. Therefore $|A \cap V| \leqslant q^{3}$. Now $A_{1} \leqslant\left\langle x\left(r_{1}, t\right) x\left(r_{3}, c t\right)\right|$ $\left.t \in F, c=t_{3} t_{1}^{-1}\right\rangle$ leads to $|A| \leqslant q^{4}$ and a contradiction. Suppose then that $a_{1}=$ $x\left(r_{1}, d\right) x\left(r_{3} b d\right) \in A_{1}$ with $d \neq 0, b \neq c$. Then $C_{V},\left(A_{1}\right) \leqslant C_{V^{\prime}}(\mathrm{a}) \cap C_{V},\left(a_{1}\right)$. But

$$
C_{V^{\prime}}(a)=\left\langle x^{\prime}\left(r_{1}+r_{2}+r_{3}, t\right) x^{\prime}\left(r_{2}+2 r_{3}, \pm 2 c t\right), X^{\prime}\left(r_{1}+r_{2}+2 r_{3}\right) \mid t \in F\right\rangle,
$$

where the sign depends only on the structure constants. Therefore $\operatorname{dim} C_{V},\left(A_{1}\right) \leqslant 1$ which implies $|A \cap V| \leqslant q^{2}$ and $|A| \leqslant q^{4}$-a contradiction. The supposition that $a_{1}=x\left(r_{3}, d\right) \in A_{1}$ with $d \neq 0$ meets a similar fate. This leaves $A_{1} \leqslant X$.

Now $C_{V},\left(A_{1}\right)=\left\langle X^{\prime}(r) \mid r \geqslant r_{1}+r_{2}\right\rangle$ and so $A \cap V \leqslant\left\langle X(r) \mid r \geqslant r_{1}+r_{2}\right\rangle .\left|A_{1}\right| \leqslant q$ and $|A|>q^{4}$ imply $|A \cap V|>q^{3}$. Let $a_{1} \in A_{1}$. Then $a_{1} v \in A$ for some $v \in V$. Let $v=v_{1} w$ 
where $v_{1} \in\left\langle X(r) \mid r \geqslant r_{2}, r \neq r_{1}\right\rangle$ and $w \in\left\langle X(r) \mid r \geqslant r_{1}+r_{2}\right\rangle$. Let $v_{2} \in A \cap V$. Now1 = $\left[a_{1} v_{1} w, v_{2}\right]=\left[v_{1}, v_{2}\right]$ for all $v_{2} \in A \cap V$. If $V_{1}=\left\langle X(r) \mid r \geqslant r_{1}+r_{2}\right\rangle$ then $V_{1}$ is the natural vector space for $\left\langle X(r) \mid r \in \Phi_{L}\right\rangle \cong B_{2}(q)$ where $L=\left\{r_{2}, r_{3}\right\}$. Now $\operatorname{dim} C_{V_{1}}\left(v_{1}\right)>3$ since $|A \cap V|>q^{3}$. By exactly the same argument as that of Lemma $5.5 \operatorname{dim} C_{V_{1}}\left(v_{1}\right)>3$ forces $v_{1}=1$. Hence $A \leqslant B-$ a contradiction. This completes the proof of Theorem 5.1 .

ProOF of TheOREM 5.2. The proof will consist of a long series of lemmas. Firstly

$$
y=\left\langle X\left(r_{3}\right), X\left(r_{4}\right), X\left(r_{3}+r_{4}\right), X\left(r_{3}+2 r_{4}\right)\right\rangle .
$$

LEMMA. 5.6. The representatives of the conjugacy classes of $Y$ are as follows:

(a) 1, one class,

(b) $x\left(r_{3}+2 r_{4}, a\right), a \neq 0, q-1$ classes,

(c) $x\left(r_{3}, b\right), b \neq 0, q-1$ classes,

(d) $x\left(r_{3}+r_{4}, c\right), c \neq 0, q-1$ classes,

(e) $x\left(r_{4}, d\right), d \neq 0, q-1$ classes,

(f) $x\left(r_{3}, b\right) x\left(r_{3}+2 r_{4}, a\right), a, b \neq 0,(q-1)^{2}$ classes,

(g) $x\left(r_{3}, b\right) x\left(r_{4}, d\right), b, d \neq 1,(q-1)^{2}$ classes.

Proof. This can be gleaned from a reading of Srinavasan (1968) or onecan compute the result by hand.

Let $A \in \mathrm{Ab}(U)$. Then $|A| \geqslant|B|=q^{7}$. As usual $(A) \varphi=A_{1} \leqslant X \times Y$ and $|A|=$ $\left|A_{1}\right||A \cap V|$. By Theorem $2.5 Y$ has a unique abelian subgroup of largest order $q^{3}$. Hence $\left|A_{1}\right| \leqslant q^{4}$. On the other hand, by Lemma 5.4 we have $|A \cap V| \leqslant q^{6}$ so that $\left|A_{1}\right| \geqslant q$.

Lemma. 5.7. $A_{1} \leqslant Y$ or $A_{1}=X$.

Proof. If $A_{1} \leqslant X$ then $\left|A_{1}\right| \geqslant q$ forces $A_{1}=X$. We will suppose that $A_{1}$ is neither contained in $X$ nor in $Y$ and obtain a contradiction. Then there exists $x, y \neq 1$ s.t. $x \in X, y \in Y$ and $x y \in A_{1}$.

Suppose $q^{2} \geqslant\left|A_{1}\right|>q$. We claim that $\left|Y \cap A_{1}\right|>1$. This we see by considering the projection epimorphism of $X \times Y$ onto $X$. Restricting to $A_{1}$, this has kernel $Y \cap A_{1}$, image contained in $X$ and so $\left|Y \cap A_{1}\right||X| \geqslant\left|A_{1}\right|$. Therefore $\left|Y \cap A_{1}\right| \geqslant\left|A_{1}\right| /|X|>1$ since $\left|A_{1}\right|>q$. Let $y_{1} \in Y \cap A_{1}, y_{1} \neq 1$. Now

$\operatorname{dim} C_{V},\left(A_{1}\right) \leqslant \operatorname{dim} C_{V},\left(\left\langle x y, y_{1}\right\rangle\right) \leqslant \operatorname{dim} C_{W}\left(y_{1}\right) \leqslant \operatorname{dim} W-2=3$ in this case.

Here we have used both Lemma 3.3 and Lemma 5.5. Thus $|A \cap V| \leqslant q^{4}$ and $|A| \leqslant q^{6}$ which is a contradiction.

Suppose now that $q^{4} \geqslant\left|A_{1}\right|>q^{2}$. Using the projection of $X \times Y$ on $X$ we get that $\left|A_{1} \cap Y\right|>q$. Now $\left|\left(A_{1} \cap Y\right) \times C_{W}\left(A_{1} \cap Y\right)\right| \leqslant q^{4}$ by Theorem 5.1. $\left|A_{1} \cap Y\right|>q$ and $C_{W}\left(A_{1} \cap Y\right)$ a vector space over $F$ force $\left|C_{W}\left(A_{1} \cap Y\right)\right| \leqslant q^{2}$. Now $x y \in A_{1}$ and 
$A_{1} \cap Y \leqslant A_{1}$ imply $\operatorname{dim} C_{Y},\left(A_{1}\right) \leqslant \operatorname{dim} C_{W}\left(A_{1} \cap Y\right) \leqslant 2$. Hence $|A \cap V| \leqslant q^{3}$ and so $\left|A_{1}\right|=q^{4}$. This forces $A_{1}=X \times E$ where $E$ is the unique element of $\mathrm{Ab}(Y)$. One checks that $\left|C_{V},\left(A_{1}\right)\right|=q$ in this case and this leads us to a contradiction.

We are left with $\left|A_{1}\right|=q$. The assumption is still that $x y \in A_{1}, x \in X, y \in Y, x, y \neq 1$. $y$ cannot be conjugate to $x\left(r_{3}+r_{4}, c\right), x\left(r_{4}, d\right)$ or $x\left(r_{3}, b\right) x\left(r_{4}, d\right), b, c, d \neq 0$, since in each case $\operatorname{dim} \operatorname{ker}_{W}(y-1)^{2}<\operatorname{dim} W=5$. This implies $\left|C_{V^{\prime}}\left(A_{1}\right)\right| \leqslant q^{4}$ which leads to $|A \cap V| \leqslant q^{5}$ and $|A| \leqslant q^{6}$-a contradiction.

Suppose $y$ is conjugate in $Y$ to $x\left(r_{3}, b\right), b \neq 0$. In fact we may syppose without loss of generality for what follows that $y=x\left(r_{3}, b\right)$. Recall that the long roots of a system of type $B_{n}$ form a system of type $D_{n}$. Now the preimage $V_{1}$ in $V$ of $C_{V},(x y)$ is a direct product of $X\left(r_{1}+r_{2}+r_{3}+r_{4}\right)$ with $D_{4}$-contribution of order $q^{5}$ as in Lemma 3.6. This $D_{4}$-contribution was found to be non-abelian for odd $q$ in the proof of Lemma 3.13. Therefore $A \cap V$ is properly contained in $V$ and so $|A \cap V|<q^{6}$. Thus $|A|<q^{7}-$ a contradiction. The same argument works for $y$ conjugate to $x\left(r_{3}+2 r_{4}, a\right), a \neq 0$ or $y$ conjugate to $x\left(r_{3}, b\right) x\left(r_{3}+2 r_{4}, a\right), a, b \neq 0$. This completes the proof of Lemma 5.7.

We suppose until further notice that $A_{1} \leqslant Y$ and so $q \leqslant\left|A_{1}\right| \leqslant q^{3},|A \cap V| \leqslant q^{6}$ and $q^{7} \leqslant|A| \leqslant q^{9}$.

Lemma 5.8. $\left|A_{1}\right|=q$ or $\left|A_{1}\right|=q^{2}$ are the only possibilities.

Proof. Suppose $q^{2} \geqslant\left|A_{1}\right|>q$. Then by Theorem 5.1(b) and the fact that $C_{W}\left(A_{1}\right)$ is a vector space over $F,\left|C_{W}\left(A_{1}\right)\right| \leqslant q^{2}$. This implies $\left|C_{V},\left(A_{1}\right)\right| \leqslant q^{4}$ which results in $|A \cap V| \leqslant q^{5}$. Now $|A| \geqslant q^{7}$ forces $\left|A_{1}\right|=q^{2}$.

Suppose instead that $q^{3} \geqslant\left|A_{1}\right|>q^{2}$. Again by Theorem 5.1(b) and the fact that $C_{W}\left(A_{1}\right)$ is a vector space over $F$ we get $\left|C_{W}\left(A_{1}\right)\right| \leqslant q$, Thus $|A \cap V| \leqslant q^{3}$ and so $|A| \leqslant q^{6}-$ a contradiction. Hence the lemma.

LemmA 5.9. Let $D_{1}=\left\langle X(r) \mid r \geqslant r_{3}, r \neq r_{2}\right\rangle$ and $D_{2}=\left\langle X(r) \mid r \geqslant r_{4}, r \neq r_{2}\right\rangle$. Then $A_{1} \leqslant D_{1}$ or $A_{1} \leqslant D_{2}$.

Proof. Suppose not, then there exists $y_{1} \in A_{1}$ such that $y_{1}$ is conjugate $y=$ $x\left(r_{3}, b\right) x\left(r_{4}, d\right), b, d \neq 0$. Then $\left|C_{w}\left(y_{1}\right)\right|=\left|C_{W}(y)\right|=q$. This implies $|A \cap V| \leqslant q^{2}$ which leads to a contradiction. Hence the lemma.

Lemma 5.10. If $x\left(r_{3}, b\right) \in A_{1}$ with $b \neq 0$, then $X\left(r_{3}\right) \leqslant A_{1}$.

Proof. Now $x\left(r_{3}, b\right) \in D_{1}$ and so by Lemma $5.9 A_{1} \leqslant D_{1}$. One checks that if $y \in D_{1}$ then $y$ commutes with the preimage in $V$ of $C_{V},(y)$. Since $C_{V},\left(A_{1}\right)=\cap_{y \in A_{1}} C_{V},(y)$ we have $\left[A_{1}, A \cap V\right]=1$. But $|A|=\left|A_{1}\right||A \cap V|$ implies that $A_{1} \times(A \cap V) \in \mathrm{Ab}(U)$. If $x=x\left(r_{3}, t\right) \notin A$ for some $t \neq 0$, consider the goup $\left\langle x, A_{1} \times(A \cap V)\right\rangle$. Then $\left[x, A_{1}\right]=1$ 
since $D_{1}$ is abelian and $[x, A \cap V]=1$ since $\left[x\left(r_{3}, b\right), A \cap V\right]=1$.Thus $\left\langle x, A_{1} \times(A \cap V)\right\rangle$ is an abelian group of larger order than $A$-contradiction. This completes the proof of Lemma 5.10.

Lemma 5.11. If $\left|A_{1}\right|=q$ then $A_{1}$ can only be one of the following:

(a) $X\left(r_{3}+2 r_{4}\right)$,

(b) $X\left(r_{3}\right)$,

(c) a conjugate of $X\left(r_{3}\right)$ by $x\left(r_{4}, t\right)$ for $t \in F^{*}$.

Proor. If $y \in A_{1}$ and $y$ conjugate to $x\left(r_{4}, d\right), d \neq 0$, we can suppose without loss of generality for what follows that $y=x\left(r_{4}, d\right)$. Then the preimage $V_{1}$ in $V$ of $C_{V^{\prime}}(y)=$ $\left\langle X\left(r_{1}+r_{2}\right), X\left(r_{2}\right), X(s) \mid s \geqslant r_{2}+r_{3}+2 r_{4}\right\rangle$. Clearly $\left[V_{1}, V_{1}\right]=X\left(r_{0}\right)$ while $Z\left(V_{1}\right)=$ $\left\langle X\left(r_{1}+r_{2}+r_{3}+2 r_{4}\right), X\left(r_{2}+r_{3}+2 r_{4}\right), X\left(r_{0}\right)\right\rangle$. Reasoning similar to that of Lemma 4.4 gives that $C \in \mathrm{Ab}\left(V_{1}\right)$ implies $|C|=q^{5}$. Therefore $|A \cap V| \leqslant q^{5}$ and so $|A| \leqslant q^{6}-\mathrm{a}$ contradiction. A similar argument rules out the case of $y \in A_{1}$ conjugate to $x\left(r_{3}+r_{4}, c\right)$, $c \neq 0$.

If $y \in A_{1}$ and $y$ is conjugate to $x\left(r_{3}, b\right) x\left(r_{3}+2 r_{4}, a\right), a, b \neq 0$ we may assume without loss of generality that $y=x\left(r_{3}, b\right) x\left(r_{3}+2 r_{4}, a\right)$. The preimage $V_{2}$ in $V$ of

$$
\begin{aligned}
C_{V^{\prime}}(y)=\langle & x\left(r_{1}+r_{2}+r_{3}, t_{1}\right) x\left(r_{1}+r_{2}+r_{3}+2 r_{4}, \pm c t_{1}\right), X\left(r_{1}+r_{2}+r_{3}+r_{4}\right), \\
& x\left(r_{2}+r_{3}, t_{2}\right) x\left(r_{2}+r_{3}+2 r_{4}, \pm c t_{2}\right), X\left(r_{2}+r_{3}+r_{4}\right), \\
& X(s)\left|s \geqslant r_{2}+2 r_{3}+2 r_{4}, t_{1}, t_{2} \in F, c=a b^{-1}\right\rangle,
\end{aligned}
$$

where the signs depend on the structure constants. Clearly $\left[V_{2}, V_{2}\right]=X\left(r_{0}\right)$. Further since the long roots of a system of type $B_{n}$ form a system of type $D_{n}$, the proof of Lemma 3.13 guarantees that $x\left(r_{1}+r_{2}+r_{3}, t_{1}\right) x\left(r_{1}+r_{2}+r_{3}+2 r_{4}, \pm c t_{1}\right)$ and $x\left(r_{2}+r_{3}, t_{2}\right) x\left(r_{2}+r_{3}+2 r_{4}, \pm c t_{2}\right)$ do not commute thus ensuring that $Z\left(V_{2}\right)=$ $\left\langle X(s) \mid s \geqslant r_{2}+2 r_{3}+2 r_{4}\right\rangle$. As for $V_{1}, C \in \mathrm{Ab}\left(V_{2}\right)$ implies $|C|=q^{5}$. This leads to $|A| \leqslant$ $q^{6}-$ a contradiction.

If $y \in A_{1}$ and $y$ is conjugate to $x\left(r_{3}, b\right), b \neq 0$, we may assume $y=x\left(r_{3}, b\right)$. Lemma 5.10 forces $X\left(r_{3}\right) \leqslant A_{1}$ and $\left|A_{1}\right|=q$ forces equality. Any element conjugate in $Y$ to $x\left(r_{3}+2 r_{4}, a\right)$ is of course equal to $x\left(r_{3}+2 r_{4}, a\right)$ since $x\left(r_{3}+2 r_{4}, a\right) \in Z(Y)$. If $x\left(r_{3}+2 r_{4}, a\right) \in A_{1}$ for $a \neq 0$, then by an argument similar to that of Lemma 5.10 we get $X\left(r_{3}+2 r_{4}\right) \leqslant A_{1}$ and $\left|A_{1}\right|=q$ gives equality. By Lemma 5.9 we have considered all the elements of $Y$ to which an element of $A_{1}$ could be conjugate and so we have proved the lemma.

LEMMA 5.12. If $\left|A_{1}\right|=q^{2}$ then $A_{1}$ can only be one of the following:

(a) $\left\langle X\left(r_{3}+r_{4}\right), X\left(r_{3}+2 r_{4}\right)\right\rangle$,

(b) $\left\langle x\left(r_{4}, t\right) x\left(r_{3}+r_{4}, a t\right), X\left(r_{3}+2 r_{4}\right) \mid t \in F\right\rangle$ for some $a \in F$,

(c) $\left\langle X\left(r_{3}\right), X\left(r_{3}+r_{4}\right)\right\rangle$,

(d) a conjugate of $\left\langle X\left(r_{3}\right), X\left(r_{3}+r_{4}\right)\right\rangle$ by $x\left(r_{4}, t\right)$ for some $t \in F^{*}$. 
Proof. (a) and (b) list all abelian subgroups of order $q^{2}$ in $D_{2}$. So we concentrate on $A_{1} \leqslant D_{1}$. Suppose $y=x\left(r_{3}, b\right) \in A_{1}$ for $b \neq 0$. Then by Lemma 5.10 we have $X\left(r_{3}\right) \leqslant A_{1}$. Suppose now that $y_{1}=x\left(r_{3}+2 r_{4}, a\right) \in A_{1}, a \neq 0$. The preimage $V_{1}$ in $V$ of $C_{V^{\prime}}(y) \cap C_{V^{\prime}}(y)=\left\langle X\left(r_{1}+r_{2}+r_{3}+r_{4}\right), X\left(r_{2}+r_{3}+r_{4}\right), X(s) \mid s \geqslant r_{2}+2 r_{3}+2 r_{4}\right\rangle$. Clearly $\left[V_{1}, V_{1}\right]=X\left(r_{0}\right)$ while $Z\left(V_{1}\right)=\left\langle X(s) \mid s \geqslant r_{2}+2 r_{3}+2 r_{4}\right\rangle$. Reasoning similar to that of Lemma 4.4 gives that $C \in \mathrm{Ab}\left(V_{1}\right)$ implies $|C|=q^{4}$. This is not sufficiently large since $\left|A_{1}\right|=q^{2}$ forces $|A \cap V| \geqslant q^{5}$. Thus $y \in A_{1}$ implies $y_{1} \notin A_{1}$.

Suppose that $y$ and $y_{2}=x\left(r_{3}+r_{4}, c\right) x\left(r_{3}+2 r_{4}, a\right) \in A_{1}, a, c \neq 0$. Then the preimage $V_{2}$ in $V$ of $C_{V},(y) \cap C_{v},\left(y_{2}\right)$ equals

$$
\begin{aligned}
\left\langlex ( r _ { 1 } + r _ { 2 } + r _ { 3 } , t _ { 1 } ) x \left( r_{1}+r_{2}+\right.\right. & \left.r_{3}+r_{4}, \pm d t_{1} / 2\right), Z\left(V_{1}\right), \\
& x\left(r_{2}+r_{3}, t_{2}\right) x\left(r_{2}+r_{3}+r_{4}, \pm d t_{2} / 2\right)\left|t_{1}, t_{2} \in F\right\rangle
\end{aligned}
$$

where $d=a c^{-1}$ and the signs depend only on the structure constants. Now $Z\left(V_{2}\right)=$ $Z\left(V_{1}\right)$ and $\left[V_{2}, V_{2}\right]=\left[V_{1}, V_{1}\right]$ imply as above that if $y \in A_{1}$ then $y_{2} \notin A_{1}$. Therefore if $y \in A_{1}$ then $A_{1} \leqslant\left\langle X\left(r_{3}\right), X\left(r_{3}+r_{4}\right)\right\rangle$ and $\left|A_{1}\right|=q^{2}$ forces equality. If an element of $A_{1}$ is conjugate in $Y$ to $y$ then $A_{1}$ is conjugate to $\left\langle X\left(r_{3}\right), X\left(r_{3}+r_{4}\right)\right\rangle$ by the same element. If an element of $A_{1}$ is conjugate in $Y$ to $x\left(r_{3}+2 r_{4}, a\right), a \neq 0$, (and hence equal), a similar argument gives $A_{1}=\left\langle X\left(r_{3}+r_{4}\right), X\left(r_{3}+2 r_{4}\right)\right\rangle$.

We are reduced to considering $A_{1} \leqslant D_{1}$ containing no conjugates of $x\left(r_{3}, b\right), b \neq 0$, or of $x\left(r_{3}+2 r_{4}, a\right), a \neq 0$. One notes since, for $a \neq 0, x\left(r_{3}+2 r_{4}, a\right) \notin A_{1}$ and since $\left|A_{1}\right|=q^{2}$ then $A_{1} \$\left\langle X\left(r_{3}+r_{4}\right), X\left(r_{3}+2 r_{4}\right)\right\rangle=Y_{1}$. Since all $Y$-conjugates of $x\left(r_{3}+r_{4}, c\right), c \neq 0$, are contained in $Y_{1}$, there exists an element $y \in A_{1}$ with $y$ conjugate to $x\left(r_{3}, b\right) x\left(r_{3}+2 r_{4}, a\right), a, b \neq 0$. As usual we may assume $y=x\left(r_{3}, b\right) x\left(r_{3}+2 r_{4} a\right)$, and then by an argument similar to that of Lemma 5.10 we get

$$
\left\langle x\left(r_{3}, t\right) x\left(r_{3}+2 r_{4}, f t\right) \mid f=a b^{-1}, t \in F\right\rangle \leqslant A_{1} .
$$

If $x\left(r_{3}, t_{1}\right) x\left(r_{3}+2 r_{4}, t_{2}\right) \in A_{1}$ with $t_{2} t_{1}^{-1} \neq f$ then $x\left(r_{3}, t_{3}\right) \in A_{1}$ for $t_{3} \neq 0$ which is a contradiction. Therefore the rest of the elements of $A_{1}$ must be of the form $x=$ $x\left(r_{3}, t_{1}\right) x\left(r_{3}+r_{4}, t_{2}\right) x\left(r_{3}+2 r_{4}, t_{3}\right)$ where $t_{1}, t_{2} \neq 0$ and $x$ is not a conjugate of $x\left(r_{3}, t_{1}\right)$ or $x$ conjugate of $x\left(r_{3}+r_{4}, t\right)$ for some $t \in F^{*}$. We claim that $A_{1}$ does in fact contain a conjugate of $x\left(r_{3}+r_{4}, t\right)$ for some $t \in F^{*}$. Suppose not. Then $y_{1}=x\left(r_{3}, t_{1}\right) x\left(r_{3}+r_{4}, t_{2}\right)$ $x\left(r_{3}+2 r_{4}, t_{3}\right) \in A_{1}$ for some $t_{1}, t_{2} \neq 0$ for otherwise $\left|A_{1}\right|=q^{2}$ and $A_{1} \leqslant\left\langle x\left(r_{3}, t\right)\right.$ $x\left(r_{3}+2 r_{4}, f t\right)|t \in F\rangle$-a contradiction. Since $x\left(r_{3}, t_{1}\right) x\left(r_{3}+2 r_{4}, f t_{1}\right) \in A_{1}$ we get that $x\left(r_{3}+r_{4}, t_{2}\right) x\left(r_{3}+2 r_{4}, t_{3}-f t_{1}\right) \in A_{1}$. This element is conjugate in $Y$ to $x\left(r_{3}+r_{4}, t_{2}\right)-\mathrm{a}$ contradiction. Hence our claim.

We now assume that $x\left(r_{3}+r_{4}, t_{2}\right) \in A_{1}$ for $t_{2} \neq 0$. As before $X\left(r_{3}+r_{4}\right) \leqslant A_{1}$. We can no longer assume that $x\left(r_{3}, b\right) x\left(r_{3}+2 r_{4}, a\right) \in A_{1}$ but only that some conjugate of it is in $A_{1}$. No element of the form $x\left(r_{3}+r_{4}, t\right) x\left(r_{3}+2 r_{4}, u\right) \in A_{1}$ where $u \neq 0$ since then we get $x\left(r_{3}+2 r_{4}, u\right) \in A_{1}$ which is in contradiction to our assumption. Further $A_{1} \$\left\langle X\left(r_{3}\right), X\left(r_{3}+r_{4}\right)\right\rangle$ since $A_{1}$ contains no element conjugate to $x\left(r_{3}, b\right), b \neq 0$. Therefore $\mathrm{A}_{1}$ contains an element of the form $y_{3}=x\left(r_{3}, t\right) x\left(r_{3}+r_{4}, u\right) x\left(r_{3}+2 r_{4}, v\right)$ 
where $t, u \neq 0$ and $y_{3}$ is not a conjugate of $x\left(r_{3}, t\right)$. But then $y_{2}=x\left(r_{3}, t\right) x\left(r_{3}+2 r_{4}, v\right)$ $\in A_{1}$. Let $y_{1}=x\left(r_{3}+r_{4}, t_{2}\right)$. Then the preimage $V_{3}$ of $C_{V},\left(y_{1}\right) \cap C_{V},\left(y_{2}\right)$ in

$$
\begin{aligned}
& V=\left\langle x\left(r_{1}+r_{2}+r_{3}, u_{1}\right) x\left(r_{1}+r_{2}+r_{3}+2 r_{4}, \pm b u_{1}\right), X(s),\right. \\
& x\left(r_{2}+r_{3}, u_{2}\right) x\left(r_{2}+r_{3}+2 r_{4}, \pm b u_{2}\right)\left|s \geqslant r_{2}+2 r_{3}+2 r_{4}, u_{1}, u_{2} \in F, b=v t^{-1}\right\rangle,
\end{aligned}
$$

where the signs depend only on the structure constants. Now $q$ odd forces $V_{3}$ nonabelian as in the proof of Lemma 3.13. Hence $|A \cap V|<q^{5}$ which leads to $|A|<q^{7}$. With this final contradiction Lemma 5.12 is proved.

In Lemmas 5.7, 5.11 and 5.12 we have limited the possibilities for $A_{1}$. We will now see that all of these possiblities do occur. We ought perhaps, at this stage, to show that $A \in \mathrm{Ab}(U)$ implies $|A|=q^{7}$ by calculating and examining the preimage of $C_{V},\left(A_{1}\right)$ for each of the possible choices for $A_{1}$ but this will become apparent anyway as we determine the possibilities for $A$.

If $A_{1}=X$ then $A=B$ without further ado. Suppose then that $A_{1}=X\left(r_{3}\right)$. Then the preimage $V_{1}$ of $C_{V},\left(A_{1}\right)$ in $V$ equals

$$
\left\langle X(r) \mid r \geqslant r_{2}+r_{3}, r \neq r_{1}+r_{2}+r_{3}+2 r_{4}, r_{2}+r_{3}+2 r_{4}\right\rangle .
$$

Now $Z\left(V_{1}\right)=\left\langle X\left(r_{1}+r_{2}+r_{3}\right), X\left(r_{2}+r_{3}\right), X(r) \mid r \geqslant r_{2}+2 r_{3}+2 r_{4}\right\rangle$. Therefore if $V_{2} \in \mathrm{Ab}\left(V_{1}\right)$ then $V_{2}$ is of the form

$$
\left\langle x\left(r_{2}+r_{3}+r_{4}, b_{2} t\right) x\left(r_{1}+r_{2}+r_{3}+r_{4}, b_{3} t\right), Z\left(V_{1}\right) \mid t \in F\right\rangle
$$

for some $\left(b_{2}, b_{3}\right) \neq(0,0)$. Order considerations force $A \cap V=V_{2}$ for some $\left(b_{2}, b_{3}\right) \neq$ $(0,0)$ and the usual argument gives $A=A_{1} V_{2} . A$ then turns out to be $C\left(0, b_{2}, b_{3}\right)$. If $A_{1}=X\left(r_{3}\right)^{x\left(r_{4}, u\right)}$ then $A=C\left(0, b_{2}, b_{3}\right)^{x\left(r_{4}, u\right)}$.

If $A_{1}=X\left(r_{3}+2 r_{4}\right)$ we get in similar fashion that $A=B\left(0,0, a_{3}, a_{4}\right)$. Suppose now that $A_{1}=\left\langle X\left(r_{3}\right), X\left(r_{3}+r_{4}\right)\right\rangle$. Then the preimage $V_{3}$ of $C_{V},\left(A_{1}\right)$ in $V$ equals

$$
\left\langle X\left(r_{1}+r_{2}+r_{3}\right), X\left(r_{2}+r_{3}\right), X(r) \mid r \geqslant r_{2}+2 r_{3}+2 r_{4}\right\rangle \text {. }
$$

$V_{3}$ is abelian and $\left|V_{3}\right|=q^{5}$. Now since $A \cap V \leqslant V_{3}$ and since $\left|A_{1}\right|=q^{2}$ forces $|A \cap V| \geqslant q^{5}$ we get equality.

Let $a \in A$ and $v \in A \cap V=V_{3}$. Then $a=a_{1} v_{1}$ where $a_{1} \in A$ and $v_{1} \in V$. Then $1=$ $[a, v]=\left[a_{1}, v\right]^{v_{1}}\left[v_{1}, v\right]=\left[v_{1}, v\right]$ since $\left[A_{1}, V_{3}\right]=1$. Thus

$$
v_{1} \in C_{V}(A \cap V)=\left\langle X(r) \mid r \geqslant r_{2}+r_{3}, r \neq r_{1}+r_{2}+r_{3}+2 r_{4}, r_{2}+r_{3}+2 r_{4}\right\rangle
$$

which in turn implies

$$
v_{1}=x\left(r_{2}+r_{3}+r_{4}, t_{1}\right) x\left(r_{1}+r_{3}+r_{3}+r_{4}, t_{2}\right) v_{2}
$$

where $v_{2} \in A \cap V$. Let $b_{1}, b_{2} \in A$ where and

$$
b_{1}=x\left(r_{3}, t_{1}\right) x\left(r_{3}+r_{4}, t_{2}\right) x\left(r_{2}+r_{3}+t_{4}, a_{1} t_{2}\right) x\left(r_{1}+r_{2}+r_{3}+r_{4}, a_{2} \dot{t_{2}}\right) w_{1}
$$

$$
b_{2}=x\left(r_{3}, u_{1}\right) x\left(r_{3}+r_{4}, u_{2}\right) x\left(r_{2}+r_{3}+r_{4}, c_{1} u_{2}\right) x\left(r_{1}+r_{2}+r_{3}+r_{4}, c_{2} u_{2}\right) w_{2}
$$


where $w_{1}, w_{2} \in A \cap V$ and $t_{2}, u_{2} \neq 0$. Then since $\left[b_{1}, b_{2}\right]=1$ we get $a_{1}=c_{1}$ and $a_{2}=c_{2}$. Again let $b_{1}, b_{2} \in A$ where $b_{2}$ is as above and

$$
b_{1}=x\left(r_{3}, t_{1}\right) x\left(r_{2}+r_{3}+r_{4}, t_{2}\right) x\left(r_{1}+r_{2}+r_{3}+r_{4}, t_{3}\right) w_{1},
$$

where $w_{1} \in A \cap V$. Since $\left[b_{1}, b_{2}\right]=1$ we get $t_{2}=t_{3}=0$. Therefore $A=C\left(1, a_{1}, a_{2}\right)$.

If $A_{1}=\left\langle X\left(r_{3}\right), X\left(r_{3}+r_{4}\right)\right\rangle^{x\left(r_{4}, t\right)}$ then $A=C\left(1, a_{1}, a_{2}\right)^{x\left(r_{4}, t\right)}$. If $A_{1}=\left\langle X\left(r_{3}+r_{4}\right)\right.$, $\left.X\left(r_{3}+2 r_{4}\right)\right\rangle$ then $A=B\left(0,1, a_{3}, a_{4}\right)$ and finally if $A_{1}=\left\langle x\left(r_{4}, t\right) x\left(r_{3}+r_{4}, a t\right)\right.$, $X\left(r_{3}+2 r_{4}\right)|t \in F\rangle$ then $A=B\left(1, a, a_{3}, a_{4}\right)$. This completes the proof of Theorem 5.2.

Proof of Theorem 5.3. We use induction on $n$. Consider firstly $n=5$. Let $A \in \operatorname{Ab}(U)$. Then $|A| \geqslant\left|B\left(a_{1}, a_{2}, \ldots, a_{5}\right)\right|=q^{11}$. As usual $(A) \varphi=A_{1} \leqslant X \times Y$ and $|A|=\left|A_{1}\right||A \cap V|$. Since $Y \in \operatorname{Syl}_{p}(N)$ where $N \cong B_{3}(q)$ we get $\left|A_{1}\right| \leqslant q^{6}$ by Theorem 5.1. On the other hand by Lemma $5.4|A \cap V| \leqslant q^{8}$ and so $\left|A_{1}\right| \geqslant q^{3}$.

\section{LEMMA 5.13. $A_{1} \leqslant Y$.}

Proof. Suppose not. Then there exists $x \in X, y \in Y$ such that $x \neq 1$ and $x y \in A_{1}$. Consider the case $q^{6} \geqslant\left|A_{1}\right| \geqslant q^{5}$. The restriction of the projection of $X \times Y$ on $X$ to $A_{1}$ has kernel $A_{1} \cap Y$, and image $\leqslant X$ and so $\left|A_{1} \cap Y\right||X| \geqslant\left|A_{1}\right|$ giving

$$
\left|A_{1} \cap Y\right| \geqslant\left|A_{1}\right| /|X| \geqslant q^{4} .
$$

By Theorem $5.2 \mid\left(A_{1} \cap Y\right) \times C_{W}\left(A_{1} \cap Y \mid \leqslant q^{7}\right.$ and so $\left|C_{W}\left(A_{1} \cap Y\right)\right| \leqslant q^{3}$. Now $x y \in A_{1}$, $A_{1} \cap Y \leqslant Y$ and Lemma 3.3 give $\operatorname{dim} C_{V},\left(A_{1}\right) \leqslant \operatorname{dim} C_{W}\left(A_{1} \cap Y\right) \leqslant 3$. Hence $|A \cap V| \leqslant q^{4}$ leading to $|A| \leqslant q^{10}$ - a contradiction. A consideration of the cases $q^{5}>\left|A_{1}\right| \geqslant q^{4}$ and $q^{4}>\left|A_{1}\right| \geqslant q^{3}$ leads in each case to the contradiction $|A|<q^{10}$. Hence $A_{1} \leqslant Y$.

Lemma 5.14. The possibilities for $A_{1}$ are:

(a) $B\left(a_{1}, a_{2}, a_{3}\right),\left(a_{1}, a_{2}, a_{3}\right) \neq(0,0,0)$,

(b) $C\left(a_{1}, a_{2}\right),\left(a_{1}, a_{2}\right) \neq\left(0,0\right.$, or a conjugate by $x\left(r_{5}, t\right)$,

(c) $B_{1}=\left\langle X(r) \mid r \geqslant r_{4}+2 r_{5}, r \neq r_{2}\right\rangle$,

(d) $C_{1}=\langle X(r)| r=r_{4}, r_{3}+r_{4}$ or $\left.r_{3}+2 r_{4}+2 r_{5}\right\rangle$ or a conjugate by $x\left(r_{5}, t\right)$; where

$$
\begin{aligned}
B\left(a_{1}, a_{2}, a_{3}\right)=\langle & x\left(r_{5}, a_{1} t\right) x\left(r_{4}+r_{5}, a_{2} t\right) x\left(r_{3}+r_{4}+r_{5}, a_{3} t\right), \\
& X(s)\left|s \geqslant r_{4}+2 r_{5}, s \neq r_{2}, t \in F\right\rangle
\end{aligned}
$$

and

$$
\begin{gathered}
C\left(a_{1}, a_{2}\right)=\left\langle x\left(r_{4}+r_{5}, a_{1} t\right) x\left(r_{3}+r_{4}+r_{5}, a_{2} t\right), X(s)\right| t \in F, \\
\left.s=r_{4}, r_{3}+r_{4} \text { or } r_{3}+2 r_{4}+2 r_{5}\right\rangle
\end{gathered}
$$

Proof. Since $A_{1} \leqslant Y$ we have $q^{5} \geqslant\left|A_{1}\right| \geqslant q^{3}$. Consider the case $q^{5} \geqslant\left|A_{1}\right|>q_{4}$. By Theorem $5.2\left|A_{1} \times C_{W}\left(A_{1}\right)\right| \leqslant q^{7}$. This implies $\left|C_{W}\left(A_{1}\right)\right| \leqslant q^{2}$ since $C_{W}\left(A_{1}\right)$ is a vector space over $F$. Hence $\left|C_{V^{\prime}}\left(A_{1}\right)\right| \leqslant q^{4}$ and $|A \cap V| \leqslant q^{5}$ leading to the contradiction 
$|A| \leqslant q^{10}$. The case $q^{4}>\left|A_{1}\right|>q^{3}$ leads in similar fashion to the contradiction $|A|<q^{11}$. Hence $\left|A_{1}\right|=q^{4}$ or $\left|A_{1}\right|=q^{3}$.

Suppose $\left|A_{1}\right|=q^{4}$. Then $\left|C_{W}\left(A_{1}\right)\right| \leqslant q^{3}$. If $\left|C_{W}\left(A_{1}\right)\right|<q^{3}$ then $\left|C_{W}\left(A_{1}\right)\right| \leqslant q^{2}$ which implies $|A \cap V| \leqslant q^{5}$ and $|A| \leqslant q^{9}-\mathrm{a}$ contradiction. Hence $\left|C_{W}\left(A_{1}\right)\right|=q^{3}$ and $\left|A_{1} \times C_{W}\left(A_{1}\right)\right|=q^{7}$. Theorem 5.2 now gives $A_{1}$ as (a) or (b).

Suppose $\left|A_{1}\right|=q^{3}$. Then as above $\left|C_{W}\left(A_{1}\right)\right|=q^{4}$ and $\left|A_{1} \times C_{W}\left(A_{1}\right)\right|=q^{7}$. Theorem 5.2 again gives $A_{1}$ as (c) or (d). This completes the proof of Lemma 5.14.

If $A_{1}=B\left(a_{1}, a_{2}, a_{3}\right)$, then $A=B\left(a_{1}, a_{2}, a_{3}, a_{4}, a_{4}\right)$ for some $a_{4}, a_{5} \in F$; if $A_{1}=C\left(a_{1}, a_{2}\right)^{x\left(r_{5}, t\right)}$, then $A=C\left(a_{1}, a_{2}, a_{3}, a_{4}\right)^{x\left(r_{5}, t\right)}$ for some $a_{3}, a_{4} \in F$; if $A_{1}=B_{1}$, then $A=B\left(0,0,0, a_{4}, a_{5}\right)$ where $\left(a_{4}, a_{5}\right) \neq(0,0)$; if $A_{1}=C_{1}^{x\left(r_{5}, t\right)}$, then $A=C\left(0,0, a_{3}, a_{4}\right)^{x\left(r_{5}, t\right)}$ for $\left(a_{3}, a_{4}\right) \neq(0,0)$. All these conclusions are arrived at by calculations similar to those at the end of Theorem 5.2.

Hence Theorem 5.3 is true for $n=5$ and we now assume it true for any integer $r$ where $5 \leqslant r<n$ and consider the case of $G=B_{n}(q)$. If $A \in \mathrm{Ab}(U)$ then $|A| \geqslant\left|B\left(a_{1}, a_{2}, \ldots, a_{n}\right)\right|=q^{n(n-1) / 2+1},(A) \varphi=A_{1} \leqslant X \times Y$ and $|A|=\left|A_{1}\right||A \cap V|$. By induction and Theorem $5.2 C \in \mathrm{Ab}(Y)$ implies $|C|=\left.q^{(n-2)(n-3)}\right|^{2+1}$. Therefore $\left|A_{1}\right| \leqslant q^{(n-2)(n-3) / 2+2}$. On the other hand by Lemma $5.4|A \cap V| \leqslant q^{2 n-2}$ and so $\left|A_{1}\right| \geqslant q^{n(n-1) / 2+1-(2 n-2)}=q^{(n-2)(n-3) / 2}$.

Lemma 5.15. $A_{1} \leqslant Y$.

Proof. Suppose not. Then there exists $x \in X, y \in Y$ such that $x \neq 1$ and $x y \in A_{1}$. We claim that there exists $y_{1} \in Y \cap A_{1}, y_{1} \neq 1$. We have proved similar claims before and we take this claim as true without further ado. Now $x y, y_{1} \in A_{1}$ implies $\operatorname{dim} C_{V},\left(A_{1}\right) \leqslant \operatorname{dim} C_{W}\left(y_{1}\right) \leqslant \operatorname{dim} W-2=2 n-5$ using Lemma 3.3 and Lemma 5.5 Thus $|A \cap V| \leqslant q^{2 n-4}$.

Now $|A| \geqslant q^{n(n-1) / 2+1}$ gives $\left|A_{1}\right|=q^{(n-2)(n-3) / 2+2}$ which implies $A_{1}=X \times E$ with $E \in \mathrm{Ab}(Y)$. For each possibility for $E$ supplied by Theorem 5.2 and induction we find $\operatorname{dim} C_{V} \cdot(X \times E) \leqslant n-2$. This gives $|A \cap V| \leqslant q^{n-1}$ and so $|A| \leqslant q^{(n-2)(n-3) / 2+2} \cdot q^{n-1}=$ $q^{\left(n^{2}-3 n+8\right) / 2}<q^{n(n-1) / 2+1}$ since $n \geqslant 6$. With this contradiction $A_{1} \leqslant Y$ is forced.

Now $A_{1} \leqslant Y$ implies $q^{(n-2)(n-3) / 2} \leqslant\left|A_{1}\right| \leqslant q^{(n-2)(n-3) / 2+1}$.

Lemma. 5.16. The possibilities for $A_{1}$ are:

(a) $B\left(a_{1}, a_{2}, \ldots, a_{n-2}\right)$ for $\left(a_{1}, a_{2}, \ldots, a_{n-2}\right) \neq(0,0, \ldots, 0)$,

(b) $C\left(a_{1}, a_{2}, \ldots, a_{n-3}\right)$ for $\left(a_{1}, a_{2}, \ldots, a_{n-3}\right) \neq(0,0, \ldots, 0)$ or a conjugate by $x\left(r_{n}, t\right)$,

(c) $B_{1}=\left\langle X(r) \mid r \geqslant r_{n-1}+2 r_{n}, r \neq r_{2}\right\rangle$,

(d) $C_{1}=\left\langle X(r), X(s) \mid r \geqslant r_{n-1}, r \neq r_{n}, s \geqslant r_{n-2}+2 r_{n-1}+2 r_{n}, r, s \neq r_{2}\right\rangle$ or a conjugate by $x\left(r_{n}, t\right)$ where the definitions of $B\left(a_{1}, a_{2}, \ldots, a_{n-2}\right)$ and $C\left(a_{1}, a_{2}, \ldots, a_{n-2}\right)$ are obvious. 
PROOF. The case $q^{(n-2)(n-3) / 2}<\left|A_{1}\right|<q^{(n-2)(n-3) / 2+1}$ is ruled out in similar fashion to the case $q^{3}<\left|A_{1}\right|<q^{4}$ in Lemma 5.14.

Suppose $\left|A_{1}\right|=q^{(n-2)(n-3) / 2+1}$. Then by induction or Theorem 5.2, $\left|A_{1} \times C_{W}\left(A_{1}\right)\right|$ $\leqslant q^{(n-1)(n-2) / 2+1}$ and so $\left|C_{W}\left(A_{1}\right)\right| \leqslant q^{n-2} .\left|C_{W}\left(A_{1}\right)\right|<q^{n-2}$ leads to a contradiction as in Lemma 5.14. Thus $\left|A_{1} \times C_{W}\left(A_{1}\right)\right|=q^{(n-1)(n-2) / 2+1}$. Induction or Theorem 5.2 gives $A_{1}$ is one of (a) or (b). In similar fashion $\left|A_{1}\right|=q^{(n-2)(n-3) / 2}$ gives $A_{1}$ is one (c) or (d). This completes the proof of Lemma 5.16 .

Exactly the same argument as in the case $n=5$ completes the picture and proves Theorem 5.3.

\section{The Thompson subgroup of $U$}

We define the Thompson subgroup $J(U)$ of $U$ as $\langle A \mid A \in \mathrm{Ab}(U)\rangle$.

THEOREM 6.1 .

(a) If $G=A_{2 n+1}(q)$ then $J(U)=\left\langle X(r) \mid r \geqslant_{n+1}\right\rangle$,

(b) if $G=A_{2 n}(q)$ then $J(U)=\langle X(r)| r \geqslant r_{n}$ or $\left.r \geqslant r_{n+1}\right\rangle$,

(c) if $G=B_{2}\left(2^{m}\right)$ then $J(U)=U$,

(d) if $G=B_{2}(q), q$ odd, then $J(U)=\left\langle X(r) \mid r \geqslant r_{1}\right\rangle$,

(e) if $G=B_{n}\left(2^{m}\right), n \geqslant 3$, then $J(U)=\left\langle X(r) \mid r \geqslant r_{n}\right\rangle$,

(f) if $G=B_{3}(q), q$ odd, then $J(U)=\left\langle X(r) \mid r \geqslant r_{1}\right\rangle$,

(g) if $G=B_{4}(q), q$ odd, then $J(U)=\langle X(r)| r \geqslant r_{1}, r_{3}$ or $\left.r_{4}\right\rangle$,

(h) if $G=B_{n}(q), q$ odd and $n \geqslant 5$, then $J(U)=\langle X(r)| r \geqslant r_{n-1}$ or $\left.r \geqslant r_{n}\right\rangle$,

(i) if $G=C_{n}(q), q$ odd and $n \geqslant 3$, then $J(U)=\left\langle X(r) \mid r \geqslant r_{n}\right\rangle$,

(j) if $G=D_{4}(q)$, then $J(U)=\langle X(r)| r \geqslant r_{1}, r_{3}$ or $\left.r_{4}\right\rangle$,

(k) if $G=D_{n}(q), n \geqslant 5$, then $J(U)=\langle X(r)| r \geqslant r_{n-1}$ or $\left.r \geqslant r_{n}\right\rangle$.

ProOF. By inspection of the results in Sections 2-5.

Corollary. 6.2. If $G$ is of type $A_{n}, B_{n}, C_{n}$ or $D_{n}$ then $J(U)=\langle A| A \leqslant U, A$ abelian of maximal rank>.

ProOF. $\mathrm{Ab}(U)$ in all cases contains elementary abelian subgroups of $U$. Thus if $A \leqslant U, A$ abelian of maximal rank, then $A \in \mathrm{Ab}(U)$. Inspection of the results of Sections 2-5 then completes the proof.

Some have defined $J(U)$ as $\langle A| A \leqslant U, A$ abelian of maximal rank $\rangle$ and so Corollory 6.2 assures us that these two definitions yield the same subgroup for the Chevalley groups under consideration here. 


\section{Conclusion}

We have not considered the case of the twisted Chevalley groups ${ }^{2} A_{n}\left(q^{2}\right)$ and ${ }^{2} D_{n}\left(q^{2}\right)$ in this paper. The methods of Section 2 can be used to determine $\operatorname{Ab}(U)$ if $G={ }^{2} A_{n+1}\left(q^{2}\right)$. In this case $|\mathrm{Ab}(U)|=1$. If $G={ }^{2} D_{n}\left(2^{2 m}\right)$ the methods of Section 4 work even though $|\mathrm{Ab}(U)| \neq 1$. However the solutions for ${ }^{2} A_{2 n}\left(q^{2}\right)$, any $q$, and ${ }^{2} D_{n}\left(q^{2}\right)$, $q$ odd, demand the introduction of more geometrical methods. Because of this and the fact that the inclusion of the twisted Chevalley groups would mean even more notation we felt that these cases should be presented in a separate paper.

\section{References}

J. L. Alperin (1965), 'Large abelian subgroups of p-groups', Trans. Amer. Math. Soc. 117, 10-20.

M. J. J. Barry (1977), Parabolic subgroups of groups of Lie type (doctoral dissertation submitted to the University of Notre Dame, Indiana).

R. W. Carter (1972), Simple groups of Lie type (John Wiley and Sons, New York, 1972).

J. T. Goozeff (1970), 'Abelian p-subgroups of GL (n,p)', J. Austral. Math. Soc. 11, 257-259.

J. E. Humphreys (1972), Introduction to Lie algebras and representation theory (Springer-Verlag, Berlin, 1972).

B. Srinivasan (1968), 'The characters of the finite symplectic group $\mathrm{Sp}(4, q)$ ', Trans. Amer. Math. Soc. 131, 488-525.

R. Steinberg (1959), 'Variations on a theme of Chevalley', Pacific J. Math. 9, 875-891.

R. Steinberg (1968), Lectures on Chevalley groups (Lecture notes (mimeo), Yale University).

G. N. Thwaites (1972), 'The Abelian p- subgroups of GL $(n, p)$ of maximal rank', Bull. London. Math. Soc. 4, 313-320.

W. J. Wong (1969), 'A characterization of the finite projective symplectic groups PSp $(4, q)$ ', Trans. Amer. Math. Soc. 139, 1-35.

Department of Mathematics

Carysfort College

Blackrock, Co. Dublin

Ireland.

Author's current address:

21 Vernon Grove

Rathgar, Dublin

Ireland 